\title{
Characterization and transcript profiling of the pectin methylesterase (PME) and pectin methylesterase inhibitor (PMEI) gene families in flax (Linum usitatissimum)
}

David Pinzón-Latorre and Michael K Deyholos ${ }^{*}$

\begin{abstract}
Background: Pectin methylesterases (PMEs) catalyze the demethylesterification of homogalacturonans in the cell wall; their activity is regulated in part by pectin methylesterase inhibitors (PMEIs). PME activity may result in either rigidification or loosening of the cell wall, depending on the mode of demethylesterification. The activity of PMEs in the middle lamella is expected to affect intrusive elongation of phloem fibers, and their adhesion to adjacent cells. Length and extractability of phloem fibers are qualities important for their industrial uses in textiles and composites. As only three flax PMEs had been previously described, we were motivated to characterize the PME and PMEl gene families of flax.

Results: We identified 105 putative flax PMEs (LuPMEs) and 95 putative PMEls (LuPMEls) within the whole-genome assembly. We found experimental evidence for the transcription of 77/105 LuPMEs and 83/95 LuPMEls, and surveyed the transcript abundance of these in 12 different tissues and stages of development. Six major monophyletic groups of LuPMEs could be defined based on the inferred relationships of flax genes and their presumed orthologs from other species. We searched the LuPMEs and LuPMEls for conserved residues previously reported to be important for their tertiary structure and function. In the LuPMEs, the most highly conserved residues were catalytic residues while in the LuPMEIs, cysteines forming disulfude bridges between helices a2 and a3 were most highly conserved. In general, the conservation of critical residues was higher in the genes with evidence of transcript expression than in those for which no expression was detected.
\end{abstract}

Conclusions: The LuPMEs and LuPMEls comprise large families with complex patterns of transcript expression and a wide range of physical characteristics. We observed that multiple PMEs and PMEls are expressed in partially overlapping domains, indicative of several genes acting redundantly during most processes. The potential for functional redundancy was highlighted also by the phylogenetic analyses. We were able to identify a subset of PME and PMEls that appeared particularly relevant to fiber development, which may provide a basis for the improvement of key traits in industrial feedstocks and a better understanding of the physiological roles of PMEs and PMEls in general.

Keywords: Flax, Pectin methylesterase, Fiber, Expression analysis, Phylogenetics, PME, PMEI

\footnotetext{
* Correspondence: deyholos@ualberta.ca

Department of Biological Sciences, University of Alberta, Edmonton, AB T6G 2E9, Canada
} 


\section{Background}

Pectins are complex polysaccharides present in the plant cell wall and in the middle lamella and are dynamically modified by pectin methylesterases (PMEs). The PME gene family was first described by Richard et al. [1], and later classified in the Carbohydrate Active Enzymes database (CAZy) as class 8 of the carbohydrate esterases (EC 3.1.1.11) [2]. In current models, pectins are synthesized in the Golgi complex as highly methylesterified polymers (e.g. homogalacturonan, HG) that are secreted to the cell wall. Once in the cell wall, PMEs catalyze the demethylesterification of HG, which generates negatively charged carboxyl groups. If demethylesterification occurs on contiguous sugar residues (i.e. blockwise demethylesterification), $\mathrm{Ca}^{2+}$ bonds can form between pectin molecules, thereby rigidifying the cell wall. Conversely, if the demethylesterification occurs on non-contiguous sugars (i.e. random demethylesterification), the molecule becomes a substrate for pectin degrading enzymes, leading to cell wall loosening $[3,4]$. The activity of the PMEs is regulated by pectin methylesterase inhibitors (PMEIs) [5], which bind to the active site of the PME, generating a 1:1 complex [6,7]. PMEs are classified as either Type-1 PMEs (i.e. those with a pro-region, similar to the PMEI domain), or Type-2 PMEs (no pro-region). In Type-1 PMEs, the pro-region and the PME domain are translated as part of the same protein and then, in the Golgi complex, as a pre-requisite for secretion to the cell wall, the pro region is removed by a subtilisin-like protease [8].

The bast (phloem) fibers of flax (Linum usitatissimum) are valued industrially for their length and strength. Extraction of high quality fibers requires retting, a process by which stems are exposed to the action of microbes that degrade the middle lamella and so, facilitate separation of fibers from surrounding tissues. Flax fibers grow from the shoot apex intrusively after a very short period of coordinated growth [9]. During intrusive elongation, fibers first penetrate the middle lamella between adjacent cells, and subsequently generate new contact interfaces. Both of these processes presumably influence fiber length and the efficiency of retting, and are dependent on the activity of PMEs.

Different varieties of flax are grown for either fibers or for seeds (i.e. linseed) [10]. Although stems of linseed varieties contain fiber, these fibers are not harvested, because of relatively low fiber yield and the difficulty of retting in the environments where linseed is typically grown. A better understanding of PMEs is therefore important to the development of dual-purpose flax, in which both fibers and seeds can be utilized from a single variety.

Three PMEs have been previously characterized in flax: LuPME1, LuPME3, and LuPME5 [11-14]. These are all
Type-1 PMEs. Al-Qsous et al. [13] reported that in $2 \mathrm{dpg}$ (days past germination) hypocotyls, transcript abundance of LuPME5 is higher in the apical region, while LuPME3 transcript abundance is higher in the basal region. Also, LuPME5 has the highest transcript abundance of the three characterized LuPMEs in hypocotyls. The highest transcript abundance of LuPME3 in seedlings is in the roots [11]. Here, we expand on these studies and present an analysis of the complete family of PMEs and PMEIs in flax, based on the recently assembled whole genome sequence of the linseed variety CDC Bethune [15]. A specific objective of this research is to identify PMEs that are expressed during stages of fiber development that are likely to influence the industrially relevant properties of flax bast fibers.

\section{Methods}

\section{Annotation of PME and PMEl domain in flax and other species}

Predicted proteins that contained PME (PF01095) and/or PMEI (PF04043) domains were identified from the whole genome shotgun (WGS) assembly of flax [15] (version 1.0) using default parameters in hmmsearch/PfamScan [16]. The predicted proteins from the flax WGS assembly were also aligned to previously described PMEs and PMEIs from Arabidopsis obtained from TAIR [17], using BLASTp. All of the LuPMEs/LuPMEIs that were identified by BLASTp to Arabidopsis were also identified by HMM-alignment to the PFAM domains. Predicted flax proteins that had both a PMEI and PME domain were designated Type-1 PMEs, and proteins with a PME domain (but no PMEI domain) were designated Type-2 PMEs. Genes with questionable PFAM annotations (i.e. significant PME and/or PMEI domain but low e-value; low coverage of the domain; more than one PME or PMEI domain; an extra domain different from PME or PMEI), and genes that were adjacent on scaffolds of the WGS assembly were manually curated, which included reanalysis of their predicted gene structures by submitting their genomic sequence (i.e. the predicted gene plus $1000 \mathrm{bp}$ up and downstream) to the Augustus web server [18]. The Augustus A. thaliana gene model parameters were used for the gene reannotation, in combination with any ESTs that aligned to the prediction region (95\% identity and 90\% coverage) [19] as well as unpublished RNAseq reads (http://www.onekp.com/, version April 25 2013).

\section{PMEs and PMEls in other plants}

An hmmsearch using PFAM domains PF01095 (Pectinesterase) and PF04043 (PMEI) was conducted with default parameters on transcripts deposited in Phytozome (version 9.1). To determine the statistical significance of the presence of the PME domain, all the protein sequences that had the domain were retrieved, and these were 
searched again against PFAM using batch search. For the putative PMEIs, protein sequences that had a PMEI domain but not a PME domain were obtained and then searched again on PFAM to establish the statistical significance of the predicted domains and confirm the absence of a PME domain.

\section{Primer design for qRT-PCR}

The Universal ProbeLibrary Assay Design Center (Roche) was used to design specific primers and probes for each gene. Groups of 10 closely related genes were submitted in batches for the design of specific primers and Roche UPL probes. The specificity of primers was evaluated by BLASTn alignment of the primers against the complete predicted transcriptome and the entire genome assembly. All primer pairs were designed so at least one primer of each pair had three or more mismatches to any off-target gene, near the 3' of the primer. For those genes for which a specific primer could not be designed, a primer common to two PME or PMEI genes was used. The list of primers can be accessed in Additional file 1: Table S1.

\section{Tissues for quantitative real time PCR using a 96.96 Dynamic array}

RNA was obtained from 12 different tissues from three biological replicates. Each biological replicate was assayed independently. Five of the tissues/organs (shoot apex (SA), leaves $(\mathrm{L})$, roots $(\mathrm{R})$, early cortical peel (ECP), and early fibers (EF)) were collected from vegetative stage plants 1 month after germination; the other seven tissues (senescent leaves (SL), xylem (X), late cortical peel (LCP), late fibers (LF), flower buds (FB), flowers (F), and green bolls (B)) were collected from plants 2 months after germination, at the green capsule stage. The cortical peel, xylem, and fiber tissues were obtained from the first $15 \mathrm{~cm}$ of the plant from the hypocotyls to the top. The shoot apex tissue corresponds to the top $2 \mathrm{~cm}$ of the plant. A phenol/ chloroform based method was used for extraction of RNA, with subsequent treatment with DNAse. 5 ng of RNA were used to synthesize the cDNA for the 96.96 dynamic array (Fluidigm Corporation, CA, USA). The cDNA was tested for genomic DNA contamination by PCR using a set of primers flanking an intron.

A total of $12.5 \mathrm{ng}$ of cDNA were used for the preamplification reaction containing $50 \mathrm{~nm}$ of each primer pair (a pool consisting of 89 primers for the PME and/or PMEI genes plus 3 endogenous controls GAPDH, ETIF1, ETIF5A [20], and 1x TaqMan PreAmp Master Mix (Applied Biosystems) in a final volume of $10 \mu \mathrm{L}$. The following thermal cycles were followed: 1 cycle: $95^{\circ} \mathrm{C}$ $10 \mathrm{~min} ; 14$ cycles: $95^{\circ} \mathrm{C} 15$ seconds, $60^{\circ} \mathrm{C} 4 \mathrm{~min}$. The pre-amplified product was diluted $1: 5$ and the preamplification reaction was tested doing a pass/fail test with GAPDH endogenous control primers, verifying that the $C_{T}$ value was close to 20 .

Primer and Roche UPL probe mix ("promer") was prepared by mixing $2 \mu \mathrm{l}$ of a $20 \mu \mathrm{M}$ mix of both primers for each $1 \mu \mathrm{L}$ of the respective probe (10 $\mu \mathrm{M}$ stock). The "promer" was tested with an equimolar mixture of cDNA from all the tissues (except fiber, which are included in the cortical peels), if it did not work new primers were designed, if the new primers did not work, it was presumed to be not expressed and the primers were used regardless, and run in the 96.96 dynamic array (Fluidigm Corporation, CA, USA).

Fluidigm 96.96 control line fluid was used to prime the fluidics arrays with the 136x chip prime script. Then the appropriate inlets were loaded with the different assays and sample mixes. The three biological replicates for each tissue were each placed in three different positions on the array for three technical replicates each.

The manufacturer's protocol was followed to prepare the assay and sample mixes. The assay inlets contained a $6.5 \mu \mathrm{L}$ assay mixture containing $1 \mathrm{x}$ DA assay loading reagent and $2 \mu \mathrm{L}$ of the respective "promer" (primer + probe) mixture for each inlet. The sample inlets contained $1 \mathrm{x}$ TaqMan ${ }^{\bullet}$ Universal PCR Master Mix, No AmpErase ${ }^{\odot}$ UNG (Applied Biosystems PN 4324018), 1x DA sample loading reagent (Fluidigm PN 85000735), and $2.5 \mu \mathrm{L}$ of the respective preamplified sample.

Once the samples and assays inlets were loaded, the 136x load mix script was executed to load the samples and assays. Once loaded, the chip was moved to the Biomark instrument and the following thermal cycles were executed: 1 cycle: $95^{\circ} \mathrm{C} 10 \mathrm{~min} ; 40$ cycles: $95^{\circ} \mathrm{C}$ 15 seconds, $60^{\circ} \mathrm{C} 1 \mathrm{~min}$.

\section{Analysis of $\mathbf{9 6 . 9 6}$ dynamic array results}

Only those wells with a quality score of $\geq 0.65$ were used in further analyses. The mean of the technical replicates was calculated. Then the delta- $\mathrm{C}_{\mathrm{T}}$ was obtained by calculating the geometric mean of the endogenous controls for the given tissue/biological-replicate, and subtracting that value to the $C_{T}$ of the gene at that tissue/biologicalreplicate. Subsequently, the mean and the standard error of the delta- $C_{T}$ of the three biological replicates were calculated.

\section{EST and RNAseq data mapping}

The EST and assembled RNAseq data (Deyholos et al., manuscript in preparation) were mapped against the PME and PMEI CDS sequences, using the read mapping tool, on the CLC Genomics Workbench 6.0.1, with the default parameters, except for the length fraction (0.8), and the similarity fraction (0.9 or 0.98$)$. 
Signal peptide, transmembrane domain, and protein subcellular localization predictions

SignalP 4.0 was used to search for signal peptides [21] Transmembrane domains were predicted using TMHMM v.2.0 [22]. The protein subcellular localization was predicted using WoLF PSORT and Plant-mPLoc [23,24].

\section{Cleavage site prediction}

Proteolytic cleavage sites were predicted using a protease recognition pattern described by Pelloux et al. and Wolf et al. [4,8]. The motif [RKQ][RKEHLN][LDMI] [LMAKR] was searched in the Type-1 PME proteins using "Protein Pattern Find" at http://www.bioinformatics.org/. These sites were also identified visually on a ClustalW multiple alignment of the protein sequences of sequences in the same phylogenetic group. This allowed us to confirm the motifs found with the web tool, and also to identify possible novel cleavage recognition motifs, by comparison of the aligned sequences with known motifs.

\section{Isoelectric point}

The predicted isoelectric point of the complete and the mature proteins (i.e. after signal peptide and/or cleavage site removal) was calculated using Vector NTI 10 [25].

\section{Phylogenetic analysis}

Phylogenetic relationships among the PMEs and PMEIs from flax, Manihot esculenta, Ricinus communis, Populus trichocarpa, and Arabidopsis thaliana were inferred as follows. The PME and PMEI protein sequences from M. esculenta, $R$. communis, $P$. trichocarpa, and A. thaliana were extracted from Phytozome (version 9.1) using the PFAM identifiers PF04043 (for PMEI) and PF01095 (for PME) in a keyword-based ontology search. Alignments for the complete PMEs and PMEIs proteins of these four species plus flax, and for the LuPMEs and LuPMEIs proteins of flax alone, were constructed using MUSCLE [26]. The alignments were used to first determine the substitution model that best described the evolutionary process of each set of proteins, using ProtTest [27], and then these models were used to construct maximum likelihood trees using GARLI [28] under the CIPRES web interface [29], with 100 bootstraps and 2 searchreplicates. The result of the analysis in ProtTest showed that the model of evolution that best fit the set of genes for LuPMEs was WAG $+\mathrm{I}+\mathrm{G}+\mathrm{F}$, and the same model was obtained for the LuPMEIs. For the analysis of the PMEs and PMEIs in all the analysed species, the best model was $\mathrm{WAG}+\mathrm{G}+\mathrm{F}$. To estimate the divergence time of presumptive paralogs (Ks) we aligned the nucleotide sequences of the LuPMEs and the LuPMEIs, and then we used MEGA5 [30] to determine genetic distance, for which we used the Kimura 2-parameter model [31] with the pairwise deletion option, and then calculated the divergence time using $t=K / 2 r$, where $t$ is time, $K$ is the genetic distance, and $\mathrm{r}$ is the substitution rate, either $1.5 \times 10-8$ [32] or $8.1 \times 10-9$ [33].

\section{Conserved residues}

The presence of the most important residues for the protein activity was established based on the structural analysis done for the PME [7] and PMEI [6,7]. For PME 11 important residues were searched: six active sites with conserved aromatic residues, three catalytic residues, and two protein stabilizers. For PMEI, 33 important residues were analysed, including residues interacting with the active sites of the PME, residues responsible for disulfide bridging, and several residues responsible for maintaining the structure of the protein.

\section{Results and discussion}

\section{Annotation of LuPMEs and LuPMEls}

We identified 105 putative LuPMEs and 95 putative LuPMEIs (Additional file 2) by searching predicted transcripts of the flax whole-genome assembly (version 1.0) [15] for the PFAM domains Pectinesterase (PF01095) and PMEI (PF04043) [16]. Independent alignment of the Arabidopsis thaliana PME and PMEI families [34] to the flax genome did not identify any additional flax genes other than those identified by the PFAM domain alignment. Among the predicted LuPMEs, 60 were Type-1 (i.e. encoding both a PMEI (PF04043) and PME (PF01095) domain [3]), and 45 were Type-2 (i.e. encoding a PME domain, but no PMEI domain [3]; Figure 1). Only one of the genes (LuPME89) contained an additional PFAM domain other than a PME or PMEI domain. This was a zf-RING_2 domain (PF13639).

\section{Detection of PMEs and PMEls in other plants}

To identify putative PMEs and PMEIs in species other than flax, primary transcripts in the Phytozome v9.1 database were searched for the presence of a PME or PMEI domain. The number of predicted PMEs and PMEIs in each species was compared as a proportion of all proteins predicted for each species (Additional file 3: Figure S1). The proportion of PMEs (0.25\%) and PMEIs $(0.22 \%)$ in flax was similar to the average proportion in the Malpighiales species sampled, i.e. $0.23 \%$ and $0.16 \%$, respectively. Among the plants analyzed, Mimulus guttatus, followed by Capsella rubella had the highest proportion of PMEs $0.30 \%$ and $0.29 \%$, respectively. Meanwhile C. rubella and Arabidopsis thaliana had the highest proportion of PMEIs $0.31 \%$ and $0.27 \%$, respectively. The proportion of PMEs was diminished significantly in the grasses as compared to other angiosperms ( $\mathrm{t}$-test $\mathrm{p}<0.01$ ). On average the angiosperms (not including grasses) had $0.22 \%$ PMEs as a proportion of the total predicted gene number, while grasses had $0.12 \%$. 


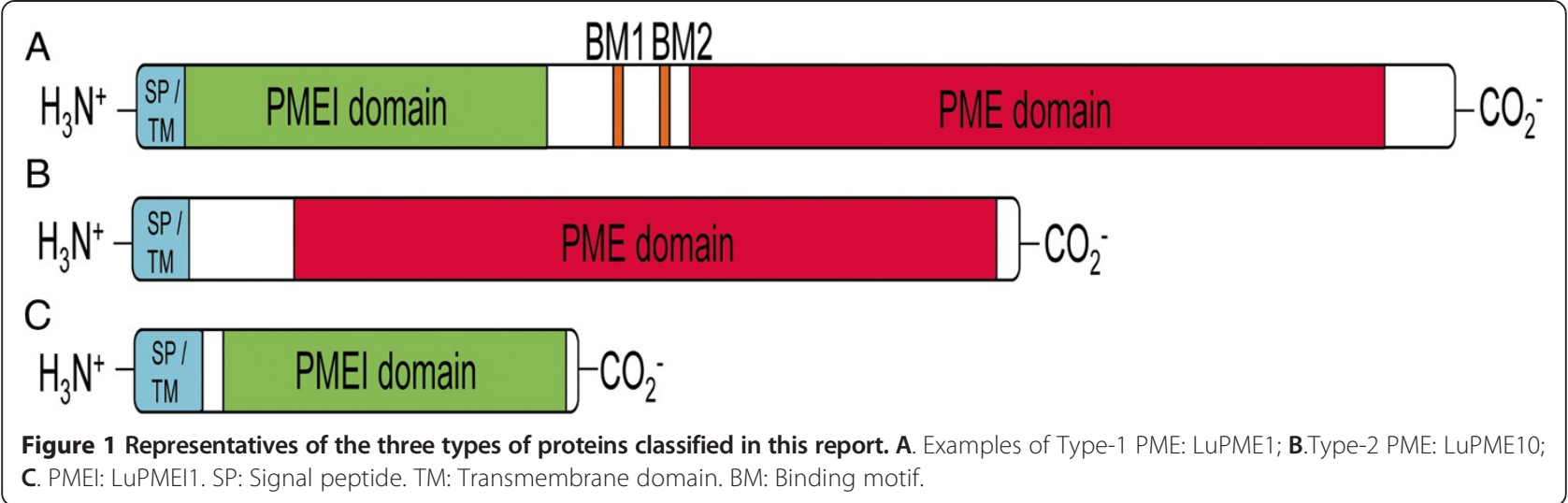

\section{Transcript expression profiling}

We examined transcript expression data to determine whether each predicted LuPME and LuPMEI was expressed, and if so, under what circumstances. Data sources for this analysis included qRT-PCR experiments (described below), published microarray data (Fenart et al. [35]), published flax ESTs (Venglat et al. [36]; NCBI), and unpublished Illumina RNAseq read data from the flax shoot apical meristem (Deyholos et al., manuscript in preparation), and from the developing flax stem (One Thousand Plants Consortium, manuscript in preparation).

\section{qRT-PCR using a 96.96 dynamic array}

We used a Fluidigm 96.96 microfluidic array to conduct qRT-PCR on 12 different tissues of flax. Five of the tissues (shoot apex (SA), leaves (L), roots (R), early cortical peel (ECP), and early fibers (EF)) were collected from vegetative stage plants 1 month after germination; the other seven tissues (senescent leaves (SL), xylem (X), late cortical peel (LCP), late fibers (LF), flower buds (FB), flowers (F), and green bolls (B)) were collected from plants 2 months after germination, at the green capsule stage. We were able to design gene-specific primers for 102 out of the 105 predicted PMEs. Transcripts of 62/102 PME genes $(60.8 \%)$ were detected in at least one of the tissues (Figure $2 \mathrm{~A}$ ), with a minimum Fluidigm 0.65 quality score and in at least 2 out of 3 biological replicates. 40/102 predicted PMEs (39.2\%) could not be detected in any of the tissues assayed by qRT-PCR. However, transcripts of $6 / 40$ of these genes could be found among public ESTs collections ( $80 \%$ coverage and $98 \%$ identity), and an additional $8 / 40$ genes could be identified among reads from RNAseq transcript profiling experiments (manuscript in preparation) (Tables 1 and 2). Finally, one of the three predicted PMEs for which qRT-PCR was not attempted was also detected by RNAseq. The unigenes assembled by Fenart et al. [35] from ESTs of a flax fiber variety were also queried. Out of the 20 unigenes (16 PMEs and 4 PMEIs) that mapped with $80 \%$ coverage and $98 \%$ identity to the predicted LuPMEs listed in Tables 1 and 2, 14 PMEs and 3 PMEIs were detected within the Fluidigm qRT-PCR array, while all were identified by either the EST collections or the RNAseq data. In total we were therefore able to find experimental evidence for the transcription of 77/105 predicted PMEs (Tables 1 and 2).

We used the same Fluidigm qRT-PCR array system to assay transcription of 94 out of 95 predicted PMEIs. $66 / 94$ genes $(70.2 \%)$ were detected in one or more tissues (Figure 2B), and 28/94 (29.8\%) PMEIs were not detected in any of the tissues. However, 17/29 of the predicted PMEIs that were not detected or assayed by qRT-PCR were identified among either public ESTs collections or in RNAseq data from developing stems (Table 3). Together, these data provide evidence that at least 83/95 (87.4\%) of the predicted PMEIs are transcribed.

Using qRT-PCR and by querying previously published and unpublished transcript databases, we were able to confirm that 77/105 and 83/95 of the predicted LuPMEs and LuPMEIs, respectively, are transcribed. The remaining genes might also be transcribed but under conditions different from those assayed to date. We note, for example, that none of the tissues surveyed to date are from plants subjected to stress, which is likely to induce PMEs that may not be otherwise transcribed. Among genes that are known to be transcribed, we found transcripts expressed in fibers and fiber bearing tissues, during either elongation (7 PMEs, 3 PMEIs), thickening (16 PMEs, 10 PMEIs), or maturation and thickening (19 PMEs, 24 PMEIs) (Table 4). These genes are primary targets for manipulation by reverse genetics, in order to develop flax feedstocks with modified fiber properties.

\section{Transcript expression patterns}

The tissue in which the largest number of expressed PMEs (48/62; 77.4\%) was detected was the flower bud. Conversely, the tissue in which the lowest number of PMEs was detected was senescent leaves (9/62; 14.5\%). The highest number of PMEIs detected was also in a reproductive tissue (flowers; 53/66 (80.3\%). Conversely, the tissue with 

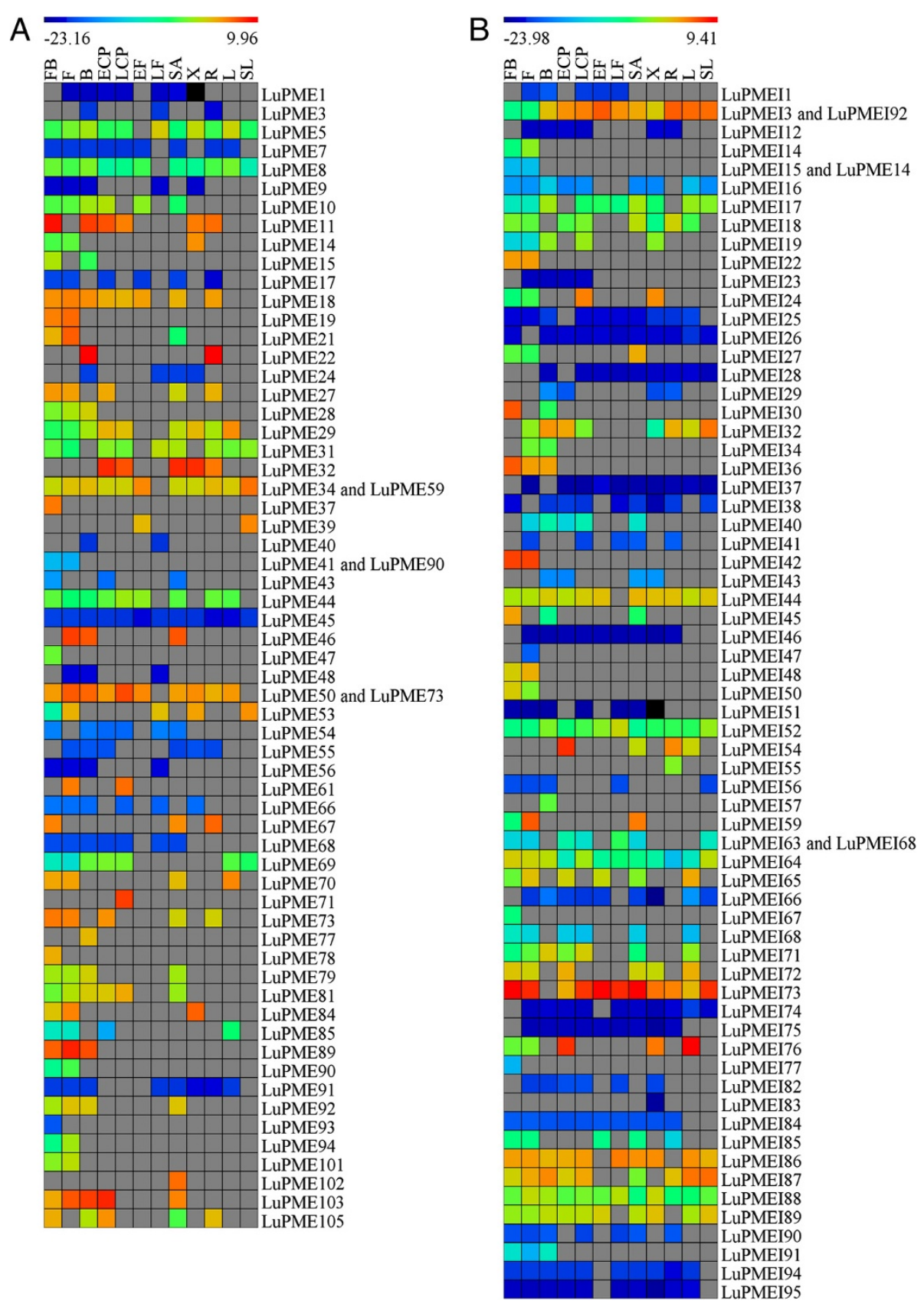

Figure 2 Heat map of transcript abundance of PMEs and PMEls in different tissues. PMEs are shown in (A) and PMEls are shown in (B). Delta- $C_{T}\left(C_{T}\right.$ of gene minus $C_{T}$ of geometric mean of the endogenous controls). The color of the cell represents transcript abundance. Gray cells indicate no transcripts were detected. When two different genes appear in the same row it means one set of primers was used as a common assay for both genes. FB: Flower buds; F: Flowers; B: Green boll; ECP: Early cortical peels; EF: Early fibers; LF: Late fibers; SA: Shoot apical meristem; X: Xylem; R: Roots; L: Leaves; SL: Senescent leaves.

the fewest detectable PMEIs was early fibers, with only 20/66 (30.3\%) (Figure 3).

We also identified PMEs and PMEIs whose transcript abundance was correlated with phloem fiber development. The transcript expression of 11 PMEs and 20 PMEIs was detected in EF, while 15 PMEs and 26 PMEIs were expressed in LF (Figure 2). 15 PMEI were expressed in both EF and LF while only one PME was expressed in both of these stages. Nine PMEs and five PMEIs were detected in EF but not LF, and conversely 13 PME and 12 PMEI were detected in LF and not EF. In general there were more PMEIs expressed in the fibers. Specifically there were more PMEIs expressed in the LF than in the EF, which might indicate that the inhibitory activity 
Table 1 Major features of type-1 LuPMEs

\begin{tabular}{|c|c|c|c|c|c|c|c|c|c|c|c|c|c|c|c|c|c|c|c|c|c|}
\hline \multirow[t]{2}{*}{ LuPME } & \multicolumn{3}{|c|}{ Mature protein } & \multicolumn{3}{|c|}{ Binding motif for cleavage } & \multicolumn{4}{|c|}{ Subcellular localization } & \multicolumn{3}{|c|}{ Conserved residues } & \multirow{2}{*}{$\begin{array}{c}\text { Tree } \\
\text { Group }\end{array}$} & \multicolumn{7}{|c|}{ Expression } \\
\hline & AA & Kda & pl & BM1 & BM2 & positions & sp & $\mathrm{tm}$ & WoIF PSORT & Plant-mPLoc & as & st & cr & & oe & $\mathrm{fl}$ & uf & ev & en & rs & rsa \\
\hline 1 & 333 & 34.9 & 8.82 & RKLL & RKLL & $203-223$ & + & - & $c h, m$ & $\mathrm{CW}$ & 6 & 2 & 3 & $\mathrm{D}$ & + & + & & & & S3 & + \\
\hline 3 & 320 & 34.4 & 9.77 & & RRLL & 235 & + & + & $c h, v$, ex & $c W$ & 6 & 2 & 3 & $\mathrm{D}$ & + & + & & & & & + \\
\hline 5 & 319 & 34.6 & 9.53 & & RRLL & 234 & - & + & pl, g_pl, er, g & $c W$ & 6 & 2 & 3 & $\mathrm{D}$ & + & + & 1 & $S, S P, F$ & 5 & All & + \\
\hline 6 & 322 & 35.5 & 7.18 & RKLL & RRVL & $202-217$ & + & - & $c h, n, p l$ & $\mathrm{CW}$ & 5 & 2 & 3 & C & + & - & & & & & + \\
\hline 7 & 322 & 35 & 6.6 & RKVA & RRLL & $235-259$ & + & - & $c h, v, n$ & $\mathrm{CW}$ & 6 & 2 & 3 & $\mathrm{D}$ & + & + & 1 & $S$ & 2 & All & + \\
\hline 8 & 364 & 40.1 & 8.66 & RKLL & RRLL & $263-286$ & - & + & $\mathrm{n}, \mathrm{cy}, \mathrm{v}, \mathrm{er}, \mathrm{m}, \mathrm{pl}$ & $\mathrm{CW}$ & 6 & 2 & 3 & B & + & + & 1 & S & 2 & S3 and 4 & + \\
\hline 9 & 322 & 35.6 & 9.6 & RRLL & RKLL & $224-242$ & + & + & ex, er, v, g, ch, n, cy & $c W$ & 5 & 2 & 3 & C & + & + & & $\mathrm{F}$ & 34 & & + \\
\hline 11 & 593 & 64.1 & 8.46 & & & & - & + & $c h, m$ & $c W$ & 6 & 2 & 3 & A & + & + & 1 & $\mathrm{ES}, \mathrm{HE}, \mathrm{TE}, \mathrm{GS}$ & 4 & All & + \\
\hline 13 & 323 & 35.3 & 10 & & RRLL & 137 & - & - & $c h$ & $\mathrm{CW}$ & 6 & 2 & 3 & $\mathrm{D}$ & - & - & & & & & \\
\hline 14 & 321 & 35.3 & 9.47 & RRLL & RKLL & $226-248$ & - & + & er, pe, ex, g, ch & $\mathrm{CW}$ & 5 & 2 & 3 & C & + & + & & $\mathrm{F}$ & 1 & SA & + \\
\hline 17 & 322 & 35.4 & 8.89 & RRLW & RRLL & $197-221$ & + & + & v, ch, ex, er, g & $\mathrm{CW}$ & 6 & 2 & 3 & D & + & + & & & & S3 and 4 & + \\
\hline 18 & 393 & 43.1 & 8.39 & RKLR & & 207 & + & - & $c h, e x, g, n, c y, p l, v, e r$ & $c W$ & 5 & 2 & 3 & D & + & + & & & & S3 and 4 & + \\
\hline 19 & 331 & 36.4 & 8.33 & RKLL & RKLL & 163-187 & - & - & $c y, n$ & $\mathrm{CW}$ & 6 & 2 & 3 & $\mathrm{D}$ & + & + & & & & & \\
\hline 22 & 324 & 35.8 & 8.8 & & RRKL & 613 & - & - & $n, c y, v$ & $\mathrm{CW}$ & 6 & 2 & 3 & D & + & + & 2 & GE & 1 & all & + \\
\hline 23 & 334 & 36.8 & 7.06 & & & & + & + & $c h, n, e x, m, p l, v$ & $\mathrm{CW}$ & 6 & 2 & 3 & D & - & - & & & & & \\
\hline 30 & 291 & 32 & 9 & RRLW & RRLL & $232-258$ & - & + & $v, c h, g, n, p l$ & $\mathrm{CW}$ & 5 & 1 & 2 & $\mathrm{D}$ & + & - & & & 4 & All & + \\
\hline 31 & 323 & 35.6 & 8.21 & RKLK & & 195 & + & + & $c h, e x, n, p l, v, e r$ & $c W$ & 6 & 2 & 3 & D & + & + & 1 & & & S3 and 4 & \\
\hline 32 & 386 & 41.2 & 9.51 & & & & - & + & $\mathrm{m}, \mathrm{ch}$ & $\mathrm{cW}$ & 1 & 0 & 0 & A & + & + & & & & S3 and 4 & + \\
\hline 36 & 534 & 58.9 & 8.97 & & & & + & - & $v, c h, n, g$ & $\mathrm{CW}$ & 6 & 2 & 3 & $\mathrm{D}$ & + & - & & & & SA & + \\
\hline 37 & 398 & 45.2 & 5.93 & RRLL & RRLL & $246-283$ & - & + & $c h, n, e x, v, e r, g$ & $\mathrm{CW}$ & 6 & 2 & 3 & C & + & + & & & & & \\
\hline 38 & 473 & 52.2 & 6.41 & & & & + & - & ex, ch, v, n, cy, pl & $c W$ & 6 & 2 & 3 & D & + & - & 1 & CE,ME & 3 & & \\
\hline 43 & 297 & 32.6 & 9.49 & RRML & RKLL & $241-273$ & - & + & er, ch, cy, v, ex & $\mathrm{CW}$ & 6 & 2 & 3 & C & + & + & & & & & \\
\hline 44 & 414 & 46 & 7.74 & & RKLL & 103 & - & - & n, cy & $\mathrm{CW}$ & 6 & 2 & 3 & D & + & + & & TS & 1 & all & + \\
\hline 45 & 318 & 33.5 & 8.6 & & RELL & 195 & + & + & $c h, c y, v, p e$ & $\mathrm{CW}$ & 5 & 2 & 3 & $\mathrm{D}$ & + & + & & TS & 2 & S3 and 4 & + \\
\hline 46 & 324 & 35.8 & 9.67 & RRLL & RRLL & $263-292$ & - & + & $n, e r, c y, c t, c h, m, v$ & $\mathrm{CW}$ & 6 & 2 & 3 & D & + & + & & & & all & + \\
\hline 47 & 326 & 35.6 & 9.17 & RRML & RKLL & $241-273$ & - & + & $v, g, c h$ & $c W$ & 6 & 2 & 3 & C & + & + & & & & & \\
\hline 49 & 525 & 58.4 & 9.29 & & & & + & - & ex, ch, v, er & $c W$ & 6 & 2 & 3 & D & + & - & 2 & & & all & + \\
\hline 50 & 427 & 46 & 9.47 & & & & + & - & $c h, n$ & $c W$ & 4 & 1 & 1 & D & + & + & 1 & & & $\mathrm{SA}$ and $\mathrm{S} 3$ & + \\
\hline 51 & 359 & 39.8 & 5.21 & RRLL & & 406 & - & - & $c y, c h, n, p l$ & $c W$ & 2 & 1 & 0 & C & - & - & & & & & \\
\hline
\end{tabular}




\begin{tabular}{|c|c|c|c|c|c|c|c|c|c|c|c|c|c|c|c|c|c|c|c|c|c|}
\hline 53 & 322 & 35.4 & 9.05 & RRLL & RRLL & $213-239$ & - & - & $c y, c h$ & $\mathrm{cW}$ & 5 & 2 & 3 & $C$ & + & + & & $F$ & 5 & & \\
\hline 54 & 533 & 58.9 & 9.79 & RRLL & RRLL & $253-281$ & - & + & $n, c y, e r$ & $\mathrm{cw}, \mathrm{n}$ & 6 & 2 & 3 & $\mathrm{D}$ & + & + & 1 & $E S, S, F$ & 3 & all & + \\
\hline 56 & 322 & 35.6 & 9.61 & RRLL & RKLL & $222-240$ & + & + & ex, er, v, g, ch, n & $\mathrm{cW}$ & 5 & 2 & 3 & $C$ & + & + & & $\mathrm{F}$ & 37 & & \\
\hline 62 & 334 & 36 & 9.94 & & RRLL & 217 & + & + & $c h, n, e x, v, c y$ & $\mathrm{cW}$ & 6 & 2 & 3 & $\mathrm{D}$ & - & - & & & & & \\
\hline 63 & 518 & 57.6 & 9.54 & & & & + & + & ch, ex, er, n, cy, m & $\mathrm{cW}$ & 6 & 2 & 3 & $\mathrm{D}$ & - & - & & & & & \\
\hline 64 & 322 & 35.9 & 10.32 & & RKVL & 358 & - & - & ch, n & cW & 6 & 2 & 3 & $\mathrm{D}$ & - & - & & & & & \\
\hline 65 & 319 & 34.3 & 9.01 & & RRLL & 187 & - & - & $\mathrm{n}, \mathrm{ch}, \mathrm{cy}, \mathrm{v}$ & $\mathrm{cW}$ & 6 & 2 & 3 & $\mathrm{D}$ & - & - & & & & & \\
\hline 66 & 348 & 38.9 & 5.87 & RRLL & RRML & $265-289$ & - & + & cy, cy_pe, pe, er, pl & $\mathrm{CW}$ & 5 & 2 & 3 & C & + & + & & $\mathrm{F}$ & 4 & & \\
\hline 70 & 387 & 42.7 & 7.71 & RKLR & & 204 & + & - & $c h, n$ & $\mathrm{cW}$ & 6 & 2 & 3 & $\mathrm{D}$ & + & + & & & & all & + \\
\hline 71 & 529 & 58.6 & 5.92 & & & & + & - & $c h, n, e x, v, m$ & $\mathrm{cW}$ & 6 & 2 & 3 & $\mathrm{D}$ & + & + & & & & & + \\
\hline 72 & 407 & 46.1 & 5.57 & RRLL & RRLL & $318-350$ & - & - & $\mathrm{n}, \mathrm{cy}, \mathrm{ch}$ & $\mathrm{CW}$ & 6 & 2 & 3 & $C$ & + & - & & $\mathrm{F}$ & 10 & & + \\
\hline 73 & 223 & 24.6 & 9.03 & & RKLL & 250 & + & - & ch, n & $c W$ & 6 & 2 & 3 & $D$ & + & + & 1 & & & all & + \\
\hline 74 & 526 & 58.6 & 9.29 & & & & + & - & ch, ex, v, er, g & $\mathrm{cW}$ & 6 & 2 & 3 & D & + & - & & & & all & + \\
\hline 75 & 327 & 36.1 & 9.92 & & GRLL & & + & + & $c h, v, n$ & $\mathrm{cW}$ & 6 & 2 & 3 & $\mathrm{D}$ & - & - & & & & & \\
\hline 76 & 335 & 36.8 & 8.76 & & RRLL & 224 & + & - & ex, ch, v & CW & 6 & 1 & 3 & $D$ & - & - & & & & & \\
\hline 78 & 400 & 45.4 & 6.12 & RRLL & RRLL & $227-258$ & + & + & $c h, e x, v, g, n$ & $c W$ & 6 & 2 & 3 & C & + & + & & & & & \\
\hline 79 & 536 & 59 & 9.04 & & & & + & - & $\mathrm{v}, \mathrm{ch}, \mathrm{n}$ & $\mathrm{cW}$ & 6 & 2 & 3 & D & + & + & & & & all & + \\
\hline 80 & 327 & 36.1 & 9.68 & & & & + & + & $c h, v$ & $\mathrm{CW}$ & 6 & 2 & 3 & D & - & - & & & & & \\
\hline 81 & 327 & 36 & 9.13 & & GRLL & & + & - & ex, er, ch, v & $\mathrm{cW}$ & 6 & 2 & 3 & $\mathrm{D}$ & + & + & & & & & \\
\hline 82 & 334 & 37.2 & 9.49 & RRLL & REYL & $242-253$ & - & + & cy, cy_n, ch, n & $\mathrm{cW}$ & 6 & 2 & 3 & B & - & - & & & & & \\
\hline 83 & 322 & 35.4 & 8.95 & RRLL & RKLL & $237-260$ & - & + & er, ex, g, ch, cy, v & $\mathrm{cW}$ & 5 & 2 & 3 & C & + & - & & $\mathrm{F}$ & 16 & & \\
\hline 84 & 547 & 60.7 & 5.25 & & & & - & + & $c h, p l, n$ & $\mathrm{CW}$ & 6 & 2 & 3 & A & + & + & & & & & \\
\hline 85 & 318 & 33.4 & 7.84 & & RKLL & 203 & + & + & $c h, c y, c y \_n, v, p e$ & $\mathrm{cW}$ & 5 & 2 & 3 & $\mathrm{D}$ & + & + & & & & S3 and 4 & \\
\hline 86 & 330 & 37 & 9.52 & RRLL & REYL & $241-252$ & - & + & $c y, c y \_n, n, c h, p l, v$ & $\mathrm{CW}$ & 5 & 2 & 3 & $B$ & - & - & & & & & \\
\hline 91 & 321 & 35.2 & 9 & RKLL & RRLL & $274-301$ & - & + & n, cy, cy_pe, v, er, ct & $c W$ & 6 & 2 & 3 & B & + & + & 1 & $\mathrm{~F}$ & 1 & all & + \\
\hline 92 & 331 & 36.3 & 7.82 & & RRLL & 267 & + & + & $\mathrm{pl}, \mathrm{er}, \mathrm{v}, \mathrm{n}$ & $\mathrm{cW}$ & 6 & 2 & 3 & D & + & + & & S & 1 & all & + \\
\hline 93 & 347 & 37.7 & 5.16 & RRLL & RRFL & $246-272$ & - & + & cy, ex, pe & $\mathrm{cW}$ & 3 & 2 & 3 & C & + & + & & F & 1 & & + \\
\hline 95 & 323 & 34.8 & 9.47 & & RRLL & 214 & + & + & ex, v, ch, g, pl & $\mathrm{cW}$ & 6 & 2 & 3 & D & - & - & & & & & \\
\hline
\end{tabular}


Table 1 Major features of type-1 LuPMEs (Continued)

\begin{tabular}{|c|c|c|c|c|c|c|c|c|c|c|c|c|c|c|c|c|c|c|}
\hline 96 & 327 & 36.6 & 10.22 & & RKVL & 154 & - & - & n, cy, pl, ct_pl, ch, ct & $\mathrm{CW}$ & 6 & 2 & 3 & D & - & - & & \\
\hline 97 & 402 & 45.1 & 10.48 & & RRVL & 228 & + & + & $v, g, n, p l, e x$ & $c w$ & 6 & 2 & 3 & D & - & - & & \\
\hline 99 & 324 & 36.2 & 8.24 & RRLL & RRML & $277-301$ & - & + & $c y, e r, c h, p l, v$ & $\mathrm{cW}$ & 5 & 2 & 3 & C & + & - & $\mathrm{F}$ & 10 \\
\hline
\end{tabular}

LuPME8, LuPME51, LuPME97, and LuPME22 have two PME domains. (SP): Presence of signal peptide; (TM): Presence of transmembrane domain. Subcellular localization: ch: chloroplast; cW: cell wall; cy: cytosol; er: endoplasmic reticulum; ex: extracellular/cell wall; g: Golgi apparatus; $\mathrm{m}$ : mitochondria; $\mathrm{n}$ : nuclear; pl: plasma membrane; v: vacuolar membrane; pe: peroxisome; ct: cytoskeleton. (AS): Number of conserved residues at active site (out of 6); (ST): Number of conserved stabilizer residues (out of 2). CR: Number of conserved catalytic residues (out of 3). Expression: A gene was reported as positive if the coverage with the EST or methods: (-) expression not des methods; (-) expression not detected in any of the methods. (FL) Gene expression based on qRT-PCR (Fluidign). (+) it was expressed in at least one tissue, (-) no expression; (NA) no assay done. (UF). Number of genes aligning wh ungenes reported by Fenart et al. [35], $80 \%$ coverage and $98 \%$ identity. (EV) Venglat et al. [36] ESTs, $80 \%$ coverage and $98 \%$ identity. (F) flower; (S) stem; (SP) stem peel; (ES) etiolated seedling; (L) leaf; (GE) globular embryo; ( Alignmentwith RNAseq assembled sequence RNAseq shoot apical meristem. 
Table 2 Important features of type-2 LuPMEs

\begin{tabular}{|c|c|c|c|c|c|c|c|c|c|c|c|c|c|c|c|c|c|c|}
\hline \multirow[t]{2}{*}{ LuPME } & \multicolumn{3}{|c|}{ Mature protein } & \multicolumn{4}{|c|}{ Subcellular localization } & \multicolumn{3}{|c|}{ \# of Conserved residues } & \multirow{2}{*}{$\begin{array}{l}\text { Tree } \\
\text { Group }\end{array}$} & \multicolumn{7}{|c|}{ Expression } \\
\hline & $\overline{A A}$ & Kda & $\mathrm{pl}$ & SP & TM & WoIF PSORT & Plant-mPLoc & AS & ST & CR & & $\mathrm{OE}$ & $\mathrm{FL}$ & UF & EV & EN & RS & RSA \\
\hline 2 & 192 & 21 & 4.8 & + & - & $c h, e x, v$ & $\mathrm{cW}$ & 1 & 1 & 2 & $\mathrm{E}$ & - & - & & & 0 & & \\
\hline 4 & 305 & 34.4 & 6.5 & - & - & $n, c y, m, c h, e x$ & $\mathrm{cW}$ & 5 & 1 & 3 & $\mathrm{E}$ & - & - & & & 0 & & \\
\hline 10 & 348 & 38.2 & 8.4 & + & + & $c h, e x, v, m$ & $\mathrm{CW}$ & 4 & 2 & 3 & $\mathrm{E}$ & + & + & & $E S, L, G E, H E, T E, M E$ & 12 & All & + \\
\hline 12 & 223 & 23.1 & 8.7 & - & - & $c h, n, m$ & $\mathrm{cW}$ & 2 & 0 & 2 & C & + & - & & & 0 & & + \\
\hline 15 & 267 & 29.9 & 5.7 & - & - & $\mathrm{n}, \mathrm{cy}, \mathrm{pl}$ & $c W$ & 3 & 1 & 2 & $\mathrm{E}$ & + & + & & & 0 & & \\
\hline 16 & 329 & 37.1 & 9.4 & + & - & ch & $\mathrm{cW}$ & 5 & 1 & 3 & E & + & - & & & 0 & & + \\
\hline 20 & 252 & 27.6 & 8.9 & - & - & $c y, c h, e x, n, m$ & $\mathrm{cW}$ & 5 & 1 & 3 & D & - & - & & & 0 & & \\
\hline 21 & 352 & 39.1 & 8.5 & - & + & ex, er, ch, cy, m, v & $\mathrm{cW}$ & 6 & 2 & 3 & E & + & + & & & 0 & All & + \\
\hline 24 & 414 & 45.4 & 8.3 & - & + & ex, ch, v, er & $\mathrm{cW}$ & 5 & 2 & 3 & $\mathrm{E}$ & + & + & 1 & & 0 & All & \\
\hline 25 & 319 & 34.7 & 8.5 & + & - & $c h, e x, m, v$ & $\mathrm{CW}$ & 4 & 1 & 3 & E & + & - & & & 0 & & + \\
\hline 26 & 322 & 35.4 & 9.5 & + & - & ch & $\mathrm{cW}$ & 5 & 1 & 3 & $\mathrm{E}$ & + & NA & & & 0 & all & + \\
\hline 27 & 363 & 39.7 & 8.9 & + & + & $c h, v, n, p l$ & $c W$ & 6 & 2 & 3 & E & + & + & & & 0 & SA and S4 & + \\
\hline 28 & 74 & 8.5 & 11.2 & - & - & $c y, c h, n, p l$ & cw, ch & 0 & 0 & 0 & $D$ & + & + & & & 0 & & \\
\hline 29 & 356 & 39.3 & 9.1 & + & + & $c h, v, n, p l$ & $\mathrm{cW}$ & 6 & 2 & 3 & E & + & + & & $\mathrm{F}$ & 2 & all & + \\
\hline 33 & 219 & 24.8 & 4.9 & - & - & $c y, c h, n, p e$ & $c W$ & 5 & 0 & 2 & A & - & - & & & 0 & & \\
\hline 34 & 317 & 35.5 & 7.7 & - & - & $c y, n, c h$ & $\mathrm{CW}$ & 6 & 2 & 3 & $\mathrm{E}$ & + & + & 1 & & 0 & & + \\
\hline 35 & 145 & 15.9 & 9.1 & - & - & $\mathrm{n}, \mathrm{ch}, \mathrm{cy}$ & $\mathrm{cW}$ & 3 & 0 & 1 & D & - & - & & & 0 & & \\
\hline 39 & 348 & 38.8 & 7.8 & + & + & $\mathrm{v}, \mathrm{cy}, \mathrm{ch}, \mathrm{m}$ & $c W$ & 4 & 2 & 3 & E & + & + & & & 0 & & \\
\hline 40 & 316 & 35.8 & 9 & + & - & $c h, n, m$ & $\mathrm{CW}$ & 5 & 1 & 3 & E & + & + & & TSC & 1 & & \\
\hline 41 & 340 & 37.3 & 6.3 & + & - & ex, v, er, ch, n & $\mathrm{cW}$ & 3 & 2 & 3 & E & + & + & & & 0 & & \\
\hline 42 & 216 & 24.4 & 8.4 & - & - & $\mathrm{m}, \mathrm{ch}, \mathrm{v}, \mathrm{n}$ & $\mathrm{cW}, \mathrm{n}$ & 1 & 0 & 0 & $\mathrm{E}$ & - & NA & & & 0 & & \\
\hline 48 & 379 & 40.2 & 8.7 & - & - & $c h, m, n$ & $\mathrm{cW}$ & 3 & 0 & 2 & C & + & + & & & 0 & & + \\
\hline 52 & 336 & 37.6 & 6 & - & - & $c y, e r, n$ & $\mathrm{CW}$ & 6 & 1 & 3 & A & + & - & & & 0 & & + \\
\hline 55 & 330 & 36.2 & 9 & + & - & $c h, v, e x, n$ & cW & 3 & 1 & 3 & E & + & + & & & 0 & & \\
\hline 57 & 260 & 28.8 & 6.8 & - & - & $\mathrm{n}, \mathrm{cy}, \mathrm{ct}, \mathrm{ex}$ & $c W$ & 2 & 1 & 3 & $\mathrm{E}$ & - & - & & & 0 & & \\
\hline 58 & 123 & 14.4 & 9.4 & - & - & $c y, n$ & $\mathrm{cW}$ & 3 & 0 & 1 & C & + & - & & $\mathrm{F}$ & 1 & & \\
\hline 59 & 318 & 35.6 & 7.7 & - & - & cy, n, ct, ct_pl & cW & 6 & 2 & 3 & E & + & + & & & 0 & SA & + \\
\hline 60 & 325 & 35.2 & 8.4 & + & + & ch, ex & cW & 4 & 1 & 3 & E & - & - & & & 0 & & \\
\hline 61 & 302 & 31.9 & 9.4 & - & - & $c y, c h, m, n$ & cW & 6 & 2 & 3 & D & + & + & 1 & & 0 & S3 and 4 & \\
\hline 67 & 418 & 45.9 & 5.6 & - & + & $v, g, e x, c h$ & $\mathrm{cW}$ & 4 & 2 & 3 & $\mathrm{E}$ & + & + & & & 0 & All & \\
\hline 68 & 316 & 35.1 & 9.4 & - & - & $\mathrm{m}, \mathrm{ch}, \mathrm{n}$ & $c W$ & 6 & 2 & 3 & $\mathrm{E}$ & + & + & & ES & 1 & SA & + \\
\hline 69 & 220 & 24.5 & 7.9 & - & - & $c h, n, c y$ & $c W$ & 5 & 2 & 3 & D & + & + & & & 0 & & \\
\hline
\end{tabular}


Table 2 Important features of type-2 LuPMEs (Continued)

\begin{tabular}{|c|c|c|c|c|c|c|c|c|c|c|c|c|c|c|c|c|c|c|}
\hline 77 & 318 & 35.2 & 7.7 & + & - & $c h, n$ & $\mathrm{CW}$ & 5 & 1 & 3 & $E$ & + & + & & & 0 & & + \\
\hline 87 & 348 & 38.5 & 7.3 & - & - & $c y, c t, n$ & $\mathrm{CW}$ & 3 & 2 & 3 & $E$ & - & - & & & 0 & & \\
\hline 88 & 140 & 15.5 & 8.9 & - & - & $c y, c h, m$ & $c W$ & 1 & 0 & 0 & $E$ & - & - & & & 0 & & \\
\hline 89 & 311 & 33.6 & 5.2 & - & - & $n, c h, c y, e x$ & $\mathrm{n}$ & 3 & 2 & 2 & $E$ & + & + & & & 0 & & + \\
\hline 90 & 339 & 37.1 & 6.3 & + & - & n, er, er_pl, m, pl, ch, cy & $c W$ & 3 & 2 & 3 & $E$ & + & + & & & 0 & & + \\
\hline 94 & 332 & 37.6 & 8.5 & + & - & ex, v, er, cy & $\mathrm{cW}$ & 3 & 1 & 2 & $E$ & + & + & & & 0 & & \\
\hline 98 & 354 & 40.1 & 8.1 & + & - & $c h, n, e x, c y$ & $\mathrm{CW}$ & 5 & 1 & 3 & $E$ & - & - & & & 0 & & \\
\hline 100 & 289 & 32.3 & 7 & - & - & $c y, n, c t, c h$ & $\mathrm{CW}$ & 1 & 0 & 2 & $E$ & - & NA & & & 0 & & \\
\hline 101 & 318 & 36.2 & 7.2 & + & - & ex, v, er, ch, cy & $\mathrm{CW}$ & 4 & 1 & 2 & $\mathrm{E}$ & + & + & & $\mathrm{F}$ & 3 & & \\
\hline 102 & 317 & 35.1 & 5.5 & - & - & $c y, n, c t$ & $c W$ & 5 & 2 & 3 & $E$ & + & + & & & 0 & All & + \\
\hline 103 & 359 & 39.8 & 8.9 & + & - & $c h, c y, n, m, e x$ & $\mathrm{CW}$ & 6 & 2 & 3 & $E$ & + & + & & & 0 & all & + \\
\hline 104 & 349 & 38.4 & 9.3 & - & - & m, ch_m, ch & $\mathrm{cW}$ & 4 & 1 & 3 & $E$ & - & - & & & 0 & & \\
\hline 105 & 343 & 37.6 & 9.1 & - & - & ch, cy & $C W$ & 6 & 2 & 3 & $\mathrm{E}$ & + & + & 1 & & 0 & SA & + \\
\hline
\end{tabular}

LUPME8, LuPME51, LuPME97, and LuPME22 have two PME domains. (SP): Presence of signal peptide; (TM): Presence of transmembrane domain. Subcellular localization: ch: chloroplast; cW: cell wall; cy: cytosol; er: endoplasmic reticulum; ex: extracellular/cell wall; g: Golgi apparatus; m: mitochondria; n: nuclear; pl: plasma membrane; v: vacuolar membrane; pe: peroxisome; ct: cytoskeleton. (AS): Number of conserved residues at active site (out of 6); (ST). Number of conserved stabilizer residues (out of 2). CR. Number of conserved catalytic residues (out of 3). Expression: A gene was reported as positive if the coverage with the EST or assembled RNAseq sequence was higher than $80 \%$ and the identity higher than $98 \%$. (OE) Overall expression based on all the methods assessed: $(+)$ expression was detected with at least one of the methods; (-) expression not detected in any of the methods. (FL) Gene expression based on qRT-PCR (Fluidigm): (+) it was expressed in at least one tissue; (-) no expression; (NA) no assay done. (UF): Number of genes aligning with unigenes reported by Fenart et al. [35], 80\% coverage and 98\%identity. (EV) Venglat et al. [36] ESTs, 80\% coverage and 98\%identity: (F) flower; (S) stem; (SP) stem peel; (ES) etiolated seedling; (L) leaf; (GE) globular embryo; (HE) heart embryo; (TE) torpedo embryo; (ME) mature embryo; (TS) torpedo seed coat; (GS) globular seed coat; (EP) endosperm pooled. (EN) Number of ESTs from NCBI as of April 2013. (RS): Alignment with RNAseq assembled sequences data (80\% coverage and 98\%identity) obtained from the OneKP project at different positions in the stem: (SA) shoot apical meristem; (S3): stem 3; (S4) stem 4. (RSA): RNAseq shoot apical meristem. 
Table 3 Important features of LuPMEls

\begin{tabular}{|c|c|c|c|c|c|c|c|c|c|c|c|c|c|c|c|c|c|c|c|c|c|}
\hline \multirow[t]{2}{*}{ LUPMEI } & \multicolumn{3}{|c|}{ Mature prot. } & \multicolumn{4}{|c|}{ Subcellular localization } & \multicolumn{7}{|c|}{ Conserved residues } & \multicolumn{7}{|c|}{ Expression } \\
\hline & $\overline{A A}$ & Kda & $\overline{\mathrm{pl}}$ & SP & TM & WoIF PSORT & Plant-mPLoc & * & $\S$ & $\square$ & $£ 2$ & $£ 3$ & $¥$ & P55 & $\overline{\mathrm{OE}}$ & $\mathrm{FL}$ & UF & EV & EN & RS & RSA \\
\hline 1 & 155 & 16.1 & 6.8 & + & - & ex, m, v, ch & $\mathrm{pl}$ & 10 & 4 & 4 & 1 & 0 & 4 & $\mathrm{~N}$ & + & + & & EP & 1 & & \\
\hline 2 & 174 & 18.9 & 8.6 & - & - & $c h, c y, m, n$ & $\mathrm{pl}, \mathrm{ch}$ & 8 & 2 & 2 & 0 & 2 & 2 & A & - & - & & & 0 & & \\
\hline 3 & 162 & 17.9 & 6.1 & + & - & ex, ch & pl & 6 & 3 & 2 & 1 & 2 & 4 & G & + & + & & & 0 & & + \\
\hline 4 & 113 & 12.6 & 4.9 & - & - & ch, n, m, pl, g_pl & $\mathrm{pl}, \mathrm{n}$ & 2 & 1 & 4 & 1 & 2 & 2 & - & - & - & & & 0 & & \\
\hline 5 & 159 & 17 & 4.9 & + & - & ex, ch, m & pl & 9 & 5 & 4 & 3 & 1 & 4 & $P$ & + & - & & $\mathrm{HE}$ & 1 & & \\
\hline 6 & 177 & 18.7 & 8.8 & + & + & v, pl, ex & $\mathrm{pl}$ & 11 & 5 & 4 & 2 & 1 & 4 & A & + & - & & GS & 1 & & \\
\hline 7 & 155 & 16.8 & 5.6 & + & + & ex, v, ch, n & pl & 11 & 4 & 3 & 1 & 3 & 4 & A & - & - & & & 0 & & \\
\hline 8 & 167 & 17.8 & 5.3 & - & + & ex, ch, v & $\mathrm{pl}$ & 7 & 2 & 3 & 2 & 1 & 4 & T & - & - & & & 0 & & \\
\hline 9 & 192 & 21.5 & 10 & + & + & $\mathrm{ch}$ & pl & 5 & 4 & 4 & 1 & 1 & 4 & T & - & - & & & 0 & & \\
\hline 10 & 199 & 21.2 & 5.1 & + & - & ex, ch, m, v & $\mathrm{pl}$ & 7 & 2 & 4 & 0 & 0 & 4 & $P$ & - & - & & & 0 & & \\
\hline 11 & 296 & 33.7 & 4.7 & - & - & $n$ & $n$ & 6 & 3 & 4 & 1 & 2 & 4 & $\mathrm{H}$ & - & - & & & 0 & & \\
\hline 12 & 155 & 16.7 & 8.4 & + & - & ex, ch & $\mathrm{pl}$ & 8 & 4 & 2 & 1 & 0 & 3 & A & + & + & & ES & 1 & & + \\
\hline 13 & 160 & 16.8 & 8.3 & + & - & ex, ch, v & $\mathrm{pl}$ & 11 & 4 & 2 & 1 & 0 & 4 & S & + & - & & & 0 & S4 & + \\
\hline 14 & 159 & 17.4 & 5.1 & + & - & $c h, v$, ex & pl & 11 & 3 & 4 & 2 & 1 & 4 & S & + & + & & & 0 & & \\
\hline 15 & 159 & 17.3 & 5.1 & + & - & $\mathrm{pl}, \mathrm{ch}, \mathrm{er}, \mathrm{ex}$ & $\mathrm{pl}$ & 11 & 3 & 4 & 2 & 1 & 4 & S & + & + & & & 0 & & \\
\hline 16 & 198 & 21.3 & 5.1 & + & - & ex & $\mathrm{pl}$ & 7 & 5 & 4 & 3 & 1 & 4 & I & + & + & & GS & 1 & $\mathrm{SA}$ and $\mathrm{S} 3$ & + \\
\hline 17 & 187 & 20.3 & 4.5 & + & + & ch & $\mathrm{pl}, \mathrm{n}$ & 8 & 4 & 3 & 1 & 2 & 4 & A & + & + & & $\mathrm{HE}, \mathrm{F}$ & 2 & S4 & + \\
\hline 18 & 181 & 19.5 & 8.3 & + & - & $c h, m, v$ & $\mathrm{pl}$ & 8 & 3 & 3 & 3 & 1 & 4 & A & + & + & & TS & 1 & SA & + \\
\hline 19 & 159 & 17 & 5 & + & - & ex, ch, m & $\mathrm{pl}$ & 9 & 5 & 4 & 3 & 1 & 4 & $P$ & + & + & & $\mathrm{GE}, \mathrm{F}$ & 4 & & \\
\hline 20 & 239 & 26 & 5.6 & - & + & ch, ch_m, n, m, cy & $\mathrm{pl}$ & 9 & 4 & 4 & 2 & 1 & 4 & s & + & - & & & 0 & $\mathrm{~S} 3$ and 4 & + \\
\hline 21 & 161 & 17.2 & 7.9 & + & - & $c h, e x, c y$ & pl & 11 & 3 & 4 & 1 & 2 & 4 & A & - & - & & & 0 & & \\
\hline 22 & 483 & 55.4 & 4.6 & + & - & ex, $v, g, n$ & $n$ & 5 & 3 & 4 & 1 & 2 & 4 & $P$ & + & + & & & 0 & & + \\
\hline 23 & 198 & 20.8 & 4.9 & + & - & $c h, e x, c y$ & pl & 6 & 3 & 3 & 1 & 1 & 4 & P & + & + & & $\mathrm{F}$ & 5 & & \\
\hline 24 & 180 & 19.6 & 8.9 & + & - & v, ch, ex & pl & 9 & 3 & 3 & 0 & 0 & 4 & $P$ & + & + & & $\mathrm{F}$ & 3 & & \\
\hline 25 & 172 & 18.6 & 4.9 & + & - & ex, ch & $\mathrm{pl}$ & 11 & 5 & 3 & 3 & 1 & 4 & K & + & + & & & 0 & & \\
\hline 26 & 154 & 16.2 & 5.5 & + & - & ex, m, ch & $\mathrm{pl}$ & 10 & 4 & 2 & 1 & 1 & 4 & A & + & + & & L & 1 & $\mathrm{SA}$ and $\mathrm{S} 3$ & + \\
\hline 27 & 149 & 16.4 & 4.7 & + & - & ex, v, ch & $\mathrm{pl}$ & 10 & 3 & 4 & 1 & 2 & 4 & $N$ & + & + & & & 0 & & \\
\hline 28 & 166 & 17.1 & 8.3 & + & - & ex, pl, ch, m, v & $\mathrm{pl}$ & 11 & 4 & 2 & 1 & 0 & 4 & S & + & + & & & 0 & SA & + \\
\hline 29 & 149 & 16.7 & 4.8 & + & - & v, ch, ex, er & pl & 10 & 2 & 4 & 1 & 1 & 4 & $N$ & + & + & & $E P, H E$ & 3 & & \\
\hline 30 & 149 & 16.4 & 4.6 & + & + & $e x, v, c y, m$ & $\mathrm{pl}$ & 10 & 2 & 4 & 1 & 0 & 4 & T & + & + & & EP,GS & 24 & & \\
\hline
\end{tabular}


Table 3 Important features of LuPMEls (Continued)

\begin{tabular}{|c|c|c|c|c|c|c|c|c|c|c|c|c|c|c|c|c|c|c|c|c|c|}
\hline 31 & 165 & 17.8 & 5.5 & + & - & $\mathrm{pl}, \mathrm{g}, \mathrm{ex}$, er & $\mathrm{pl}$ & 11 & 4 & 2 & 1 & 1 & 3 & K & - & - & & & 0 & & \\
\hline 32 & 155 & 16.5 & 8.4 & + & + & $c h, v, c y, n, m$ & $\mathrm{pl}$ & 10 & 4 & 3 & 1 & 0 & 4 & $\mathrm{~T}$ & + & + & & & 0 & & \\
\hline 33 & 152 & 15.9 & 5.1 & + & - & ex, ch & $\mathrm{pl}$ & 9 & 4 & 3 & 1 & 2 & 4 & A & + & - & & GS & 1 & & \\
\hline 34 & 152 & 16.2 & 4.7 & + & + & $v, \mathrm{pl}$ & $\mathrm{pl}$ & 11 & 4 & 4 & 2 & 2 & 4 & A & + & + & & & 0 & & \\
\hline 35 & 154 & 16.1 & 4.6 & + & - & ex, ch, n & $\mathrm{pl}$ & 10 & 4 & 4 & 2 & 2 & 4 & A & + & - & & & 0 & & + \\
\hline 36 & 152 & 16.3 & 4.7 & + & - & $e x, c h, c y, m$ & $\mathrm{pl}$ & 11 & 5 & 4 & 3 & 2 & 4 & A & + & + & & & 0 & & \\
\hline 37 & 167 & 17.9 & 5.4 & - & - & $c h, c y, n, c t \_n$ & $\mathrm{pl}$ & 8 & 4 & 4 & 2 & 1 & 4 & $P$ & + & + & & & 0 & & + \\
\hline 38 & 163 & 17.8 & 7.8 & + & - & ex, ch, pl & $\mathrm{pl}$ & 9 & 5 & 3 & 1 & 2 & 4 & $P$ & + & + & & & 0 & S3 & + \\
\hline 39 & 158 & 17.4 & 4.5 & - & - & $c h, e x, n$ & $\mathrm{pl}$ & 10 & 5 & 2 & 3 & 1 & 4 & K & + & - & & & 0 & & + \\
\hline 40 & 164 & 17.6 & 5.7 & + & + & v, ex, pl, m, er_pl, n & $\mathrm{pl}$ & 9 & 5 & 3 & 2 & 2 & 4 & A & + & + & 1 & $\mathrm{HE}$ & 1 & & + \\
\hline 41 & 151 & 16.1 & 5.3 & + & - & $c h, v, n, m, e x$ & $\mathrm{pl}$ & 11 & 5 & 2 & 1 & 2 & 4 & M & + & + & & $H E, G S$ & 2 & & \\
\hline 42 & 159 & 17.2 & 6.1 & + & - & $e x, c h, v, m$ & $\mathrm{pl}$ & 10 & 3 & 4 & 1 & 1 & 4 & $S$ & + & + & & & 0 & & \\
\hline 43 & 181 & 19.6 & 9.5 & + & - & $c h, v$ & $\mathrm{pl}$ & 7 & 4 & 4 & 1 & 1 & 4 & I & + & + & & & 0 & $\mathrm{SA}$ and $\mathrm{S} 3$ & + \\
\hline 44 & 187 & 19.9 & 6.8 & + & + & ex, v & $\mathrm{pl}$ & 7 & 5 & 4 & 2 & 1 & 4 & I & + & + & & & 0 & All & + \\
\hline 45 & 159 & 17.3 & 6.5 & + & - & ex, ch, n, cy & $\mathrm{pl}$ & 7 & 3 & 4 & 3 & 1 & 4 & I & + & + & & & 0 & SA & + \\
\hline 46 & 389 & 45 & 5.1 & - & - & $n$ & $n$ & 5 & 3 & 4 & 1 & 2 & 4 & $P$ & + & + & & & 0 & & + \\
\hline 47 & 188 & 20.2 & 8.4 & + & - & $e x, v, c h, m$ & $\mathrm{pl}, \mathrm{n}$ & 7 & 3 & 4 & 0 & 1 & 4 & $P$ & + & + & & & 0 & & \\
\hline 48 & 193 & 21.2 & 7.7 & + & + & $\mathrm{n}, \mathrm{ex}, \mathrm{ch}, \mathrm{cy}, \mathrm{v}$ & $\mathrm{pl}, \mathrm{n}$ & 7 & 3 & 4 & 1 & 0 & 4 & $P$ & + & + & & $\mathrm{F}$ & 2 & & \\
\hline 49 & 197 & 21.5 & 6.9 & + & + & ex, ch, n, m, v & $\mathrm{pl}, \mathrm{n}$ & 8 & 3 & 4 & 1 & 0 & 4 & $P$ & - & NA & & & 0 & & \\
\hline 50 & 467 & 50.6 & 6.5 & - & - & $\mathrm{n}, \mathrm{ch}, \mathrm{pl}, \mathrm{m}$ & $\mathrm{pl}, \mathrm{n}$ & 7 & 4 & 3 & 1 & 3 & 4 & A & + & + & & & 0 & & \\
\hline 51 & 368 & 37.4 & 4.3 & + & + & ex, ch, v, n & $\mathrm{n}$ & 8 & 4 & 2 & 1 & 2 & 4 & $P$ & + & + & & & 0 & & + \\
\hline 52 & 65 & 7.3 & 4.5 & - & - & $c y, n, c h, p l, e x$ & $\mathrm{pl}, \mathrm{n}$ & 5 & 1 & 1 & 0 & 1 & 1 & - & + & + & & & 0 & & \\
\hline 53 & 161 & 17.8 & 9 & + & + & ch, ex & $\mathrm{pl}$ & 7 & 5 & 4 & 3 & 1 & 4 & I & + & - & & $\mathrm{S}$ & 1 & & + \\
\hline 54 & 175 & 18.7 & 6.1 & + & - & $c h, m, e x$ & $\mathrm{pl}$ & 7 & 5 & 4 & 3 & 1 & 4 & I & + & + & & & 0 & All & + \\
\hline 55 & 141 & 15.3 & 5.9 & - & - & $c h, m$ & $\mathrm{cW}$ & 4 & 1 & 4 & 2 & 1 & 3 & - & + & + & & & 0 & & \\
\hline 56 & 167 & 18.8 & 8.3 & + & - & $c h, e x, c y, m, p l$ & $\mathrm{pl}, \mathrm{n}$ & 7 & 4 & 3 & 1 & 2 & 4 & D & + & + & & & 0 & & + \\
\hline 57 & 154 & 16 & 6.4 & + & + & $e x, c h, c y, m$ & $\mathrm{pl}$ & 9 & 4 & 4 & 2 & 0 & 4 & N & + & + & & GE & 1 & & \\
\hline 58 & 195 & 21.1 & 5.2 & + & - & ex, ch & $\mathrm{pl}$ & 7 & 5 & 4 & 3 & 1 & 4 & । & + & - & & & 0 & all & \\
\hline 59 & 173 & 18.9 & 4.5 & + & - & ex, ch, n & $\mathrm{pl}$ & 10 & 5 & 2 & 3 & 1 & 4 & K & + & + & & & 0 & & \\
\hline 60 & 195 & 21.7 & 9.9 & + & - & ch & $\mathrm{pl}$ & 6 & 4 & 4 & 1 & 1 & 4 & $\mathrm{~T}$ & + & - & & & 0 & & + \\
\hline
\end{tabular}


Table 3 Important features of LuPMEls (Continued)

\begin{tabular}{|c|c|c|c|c|c|c|c|c|c|c|c|c|c|c|c|c|c|c|c|c|c|}
\hline 61 & 468 & 51.7 & 8.7 & - & - & $\mathrm{n}, \mathrm{ch}, \mathrm{cy}$ & $\mathrm{pl}, \mathrm{n}$ & 7 & 4 & 3 & 3 & 1 & 3 & G & + & - & & & 0 & All & + \\
\hline 62 & 118 & 13.2 & 6.9 & - & + & $c h, c y, n, e x$ & $\mathrm{pl}$ & 4 & 1 & 3 & 2 & 3 & 2 & - & - & - & & & 0 & & \\
\hline 63 & 170 & 18.3 & 5.9 & + & - & $c h, v, e x, m$ & $\mathrm{pl}$ & 7 & 5 & 4 & 2 & 2 & 4 & V & + & + & & TE & 1 & & + \\
\hline 64 & 177 & 19.1 & 10 & + & - & ch & $\mathrm{pl}$ & 7 & 4 & 4 & 3 & 1 & 4 & I & + & + & & & 0 & SA & + \\
\hline 65 & 180 & 19.1 & 5.3 & + & - & $c h, m$ & $\mathrm{pl}$ & 6 & 5 & 4 & 3 & 1 & 4 & 1 & + & + & 1 & & 0 & all & + \\
\hline 66 & 160 & 17.8 & 7.8 & + & - & ex, ch, n, cy & $\mathrm{pl}$ & 7 & 3 & 4 & 0 & 2 & 4 & $\mathrm{~T}$ & + & + & & TS, EP & 4 & SA & \\
\hline 67 & 174 & 18.8 & 4.6 & + & - & ex, ch & $\mathrm{pl}$ & 11 & 5 & 4 & 3 & 1 & 4 & K & + & + & & & 0 & & \\
\hline 68 & 177 & 19 & 5.5 & + & - & $c h, e x, v, m$ & $\mathrm{pl}$ & 7 & 5 & 4 & 3 & 2 & 4 & V & + & + & & & 0 & & + \\
\hline 69 & 179 & 19.3 & 9.2 & + & - & $\mathrm{ch}$ & $\mathrm{pl}$ & 7 & 5 & 4 & 3 & 2 & 4 & 1 & + & - & & & 0 & & + \\
\hline 70 & 177 & 19.2 & 10.1 & + & - & ch & $\mathrm{pl}$ & 7 & 4 & 4 & 3 & 1 & 4 & 1 & + & - & & & 0 & All & + \\
\hline 71 & 183 & 19.6 & 5.7 & + & - & $c h, m$ & $\mathrm{pl}$ & 6 & 5 & 4 & 3 & 1 & 4 & 1 & + & + & & & 0 & & + \\
\hline 72 & 183 & 20 & 9.4 & + & - & ch, n & $\mathrm{pl}$ & 7 & 5 & 4 & 1 & 1 & 4 & 1 & + & + & & & 0 & & + \\
\hline 73 & 212 & 22.6 & 7.7 & + & - & $e x, m, v$ & $\mathrm{pl}$ & 7 & 5 & 4 & 2 & 1 & 4 & 1 & + & + & & & 0 & SA and S4 & + \\
\hline 74 & 159 & 17.4 & 5.5 & + & - & $c h, e x, n, c y, m$ & $\mathrm{pl}$ & 6 & 3 & 4 & 3 & 1 & 3 & 1 & + & + & & & 0 & S3 and 4 & + \\
\hline 75 & 170 & 19.1 & 9 & + & - & $\mathrm{ch}, \mathrm{pl}$ & $\mathrm{pl}, \mathrm{n}$ & 7 & 5 & 3 & 1 & 2 & 4 & D & + & + & & & 0 & S4 & + \\
\hline 76 & 228 & 25 & 9 & + & - & $c h, v, e x$ & $\mathrm{n}$ & 9 & 3 & 2 & 0 & 0 & 4 & $P$ & + & + & & $\mathrm{F}$ & 1 & & \\
\hline 77 & 220 & 23 & 4.6 & - & - & $c h, n$ & $\mathrm{pl}$ & 9 & 3 & 3 & 1 & 1 & 4 & P & + & + & & & 0 & & \\
\hline 78 & 163 & 17.7 & 5.7 & + & - & $\mathrm{pl}, \mathrm{g}, \mathrm{ex}, \mathrm{ch}$ & $\mathrm{pl}$ & 11 & 4 & 2 & 1 & 1 & 4 & K & + & - & & & 0 & SA and S3 & \\
\hline 79 & 155 & 16.6 & 6.9 & + & + & er, ex, er_pl, ch, m, pl, cy & $\mathrm{pl}$ & 10 & 4 & 3 & 1 & 0 & 4 & $\mathrm{~T}$ & + & - & & & 0 & S3 and 4 & \\
\hline 80 & 229 & 24.9 & 5.7 & + & - & ch, ex & $\mathrm{pl}, \mathrm{n}$ & 7 & 4 & 3 & 2 & 2 & 4 & A & - & - & & & 0 & & \\
\hline 81 & 184 & 19.3 & 4.8 & - & - & $c h, m, n$ & $\mathrm{pl}$ & 5 & 2 & 4 & 2 & 1 & 3 & P & + & - & 1 & & 0 & S3 & + \\
\hline 82 & 334 & 34.4 & 4.4 & + & + & ex, ch, v, n & $n$ & 8 & 4 & 2 & 1 & 2 & 4 & $P$ & + & + & & & 0 & & \\
\hline 83 & 249 & 26.9 & 4.7 & + & - & $c h, e x, v$ & $\mathrm{pl}, \mathrm{n}$ & 7 & 4 & 3 & 1 & 2 & 4 & A & + & + & & & 0 & & \\
\hline 84 & 193 & 20.5 & 4.7 & + & - & ex, ch, v & $\mathrm{pl}$ & 7 & 5 & 4 & 2 & 1 & 4 & V & + & + & & & 0 & S3 & + \\
\hline 85 & 156 & 16.9 & 8.4 & + & - & $e x, v, p l$ & $\mathrm{pl}$ & 8 & 3 & 3 & 0 & 0 & 4 & R & + & + & & & 0 & All & + \\
\hline 86 & 150 & 16.2 & 9.2 & + & + & v, ex, pl & $\mathrm{pl}$ & 10 & 2 & 4 & 0 & 1 & 4 & A & + & + & & & 0 & All & + \\
\hline 87 & 162 & 17.7 & 9 & + & - & $c h$ & $\mathrm{pl}$ & 7 & 5 & 4 & 3 & 1 & 4 & I & + & + & & & 0 & & + \\
\hline 88 & 172 & 18.3 & 5.4 & + & + & ch & $\mathrm{pl}$ & 7 & 5 & 4 & 3 & 1 & 4 & 1 & + & + & & TS & 1 & All & + \\
\hline 89 & 155 & 16.6 & 8.8 & + & + & ex, pl, v, ch, cy, m & $\mathrm{pl}$ & 10 & 2 & 4 & 0 & 1 & 4 & A & + & + & & & 0 & S3 and 4 & + \\
\hline 90 & 188 & 19.9 & 5.7 & - & - & $c h, m, c y$ & $\mathrm{pl}, \mathrm{cy}$ & 6 & 3 & 3 & 2 & 1 & 2 & P & + & + & & $\mathrm{F}$ & 1 & & + \\
\hline
\end{tabular}


Table 3 Important features of LuPMEls (Continued)

\begin{tabular}{|c|c|c|c|c|c|c|c|c|c|c|c|c|c|c|c|c|c|c|c|c|c|}
\hline 91 & 327 & 36.6 & 9.2 & - & + & $\mathrm{pl}, \mathrm{ch}, \mathrm{m}, \mathrm{er}$ & $\mathrm{pl}$ & 9 & 4 & 3 & 2 & 3 & 4 & T & + & + & & & 0 & & \\
\hline 92 & 162 & 17.9 & 7.8 & + & - & ex, ch & $\mathrm{pl}$ & 6 & 3 & 2 & 1 & 2 & 4 & G & + & + & & & 0 & all & + \\
\hline 93 & 156 & 16.5 & 4.4 & + & + & n, ex, m, er_pl, pl, er & $\mathrm{pl}$ & 11 & 5 & 4 & 2 & 2 & 4 & G & + & - & & EP & 1 & & \\
\hline 94 & 331 & 35.9 & 6.3 & - & + & n, g, pl, er_pl, cy & cy & 9 & 4 & 4 & 2 & 1 & 4 & S & + & + & 1 & TE & 1 & SA and S4 & + \\
\hline 95 & 157 & 17.3 & 5.3 & - & - & $c y, c h, n, e x$ & $\mathrm{pl}$ & 8 & 4 & 3 & 1 & 2 & 3 & $P$ & + & + & & & 0 & & + \\
\hline
\end{tabular}

(SP): presence of signal peptide; (TM): presence of transmembrane domain. Subcellular localization: ch: chloroplast; cw: cell wall; cy: cytosol; er: endoplasmic reticulum; ex: extracellular/cell wall; g: Golgi apparatus; $\mathrm{m}$; mitochondria; n: nuclear; pl: plasma membrane; v: vacuolar membrane; pe: peroxisome; ct: cytoskeleton. Number of conserved residues at each one of the following: ${ }^{*}$ ) non polar bundle-hairpin interface (out of 12 ); (\$) polar bundle-hairpin interface (out of 5); (口) Polar interacting with PME aromatic residues (out of 5); (£2) acidic patch on a2 helix (out of 3); (£3) acidic patch on a3 helix (out of 3); (¥) disulphide bridge (out of 4). Expression: A gene was reported as positive if the coverage with the EST or assembled RNAseq sequence was higher than $80 \%$ and the identity higher than $98 \%$. (OE) Overall expression based on all the methods assessed: (+) expression was detected with at least one of the methods; (-) expression not detected in any of the methods. (FL) Gene expression based on qRT-PCR (Fluidigm): (+) it was expressed in at least one tissue; (-) no expression; (NA) no assay done. (UF): Number of genes aligning with unigenes reported by Fenart et al. [35], 80\% coverage and 98\% identity. (EV) Venglat et al. [36] ESTs, 80\% coverage and 98\%identity: (F) flower; (S) stem; (SP) stem peel; (ES) etiolated seedling; (L) leaf; (GE) globular embryo; (HE) heart embryo; (TE) torpedo embryo; (ME) mature embryo; (TS) torpedo seed coat; (GS) globular seed coat; (EP) endosperm shoot apical meristem; (S3): stem 3; (S4) stem 4. (RSA): RNAseq shoot apical meristem. 
Table 4 Genes putatively associated with fiber development during elongation, thickening, and maturation processes

\begin{tabular}{|c|c|c|c|}
\hline & Gene & Expression pattern & Homologous to \\
\hline$\S$ & LUPME1 & $L-E$ & AtPME35 \\
\hline$\S$ & LUPME3 & $O-X_{i}$ L-E & PttPME1 \\
\hline$\S$ & LUPME5 & $L-E$ & \\
\hline$¥$ & LUPME7 & $O-X ; E-L$ & PttPME1 \\
\hline$¥$ & LuPME8 & E-L & \\
\hline$\S$ & LuPME9 & $L-E$ & \\
\hline$¥$ & LUPME10 & O-X; E-L; EC-LC & \\
\hline$¥$ & LuPME17 & O-X; E-L; EC-LC & \\
\hline$¥$ & LUPME18 & O-X; E-L & \\
\hline * & LUPME21 & SA-S & \\
\hline$\S$ & LuPME24 & $L-E$ & \\
\hline$¥$ & LUPME27 & O-X; EC-LC & \\
\hline$\S$ & LUPME31 & $O-X ; L-E$ & \\
\hline$\S$ & LUPME32 & OV & \\
\hline$¥$ & LuPME34 and LuPME59 & $\mathrm{E}-\mathrm{L}$ & \\
\hline$¥$ & LUPME39 & $O-X ; E-L$ & \\
\hline$\S$ & LUPME40 & $O-X ; L-E$ & \\
\hline$¥$ & LUPME43 & O-X; EC-LC & \\
\hline$¥$ & LuPME44 & $\mathrm{O}-\mathrm{X} ; \mathrm{E}-\mathrm{L}$ & \\
\hline * & $\underline{\text { LUPME46 }}$ & SA-S & \\
\hline$\S$ & LuPME48 & $O-X ; L-E$ & \\
\hline$¥$ & LUPME50 and LuPME73 & $E-L$ & \\
\hline$\S$ & LUPME53 & $L-E$ & \\
\hline$\S$ & LUPME54 & $O-X ; L-E$ & \\
\hline$¥$ & LuPME55 & EC-LC & \\
\hline$\S$ & LuPME56 & $O-X ; L-E$ & \\
\hline$\S$ & LuPME61 & O-X; LC-EC & AtPME35 \\
\hline$\S$ & LuPME66 & L-E; LC-EC & \\
\hline * & $\underline{\text { LuPME67 }}$ & SA-S & \\
\hline$\S$ & LuPME68 & $O-X_{i} L-E$ & \\
\hline$\S$ & LuPME69 & $O-X$ & \\
\hline * & LUPME70 & SA-S & \\
\hline$\S$ & LUPME71 & OV; O-X; LC-EC; LCP & \\
\hline$¥$ & LUPME73 & O-X; EC-LC & \\
\hline * & $\underline{\text { LUPME79 }}$ & SA-S & \\
\hline$\S$ & LUPME81 & $O-X$ & \\
\hline$¥$ & LuPME85 & O-X; EC-LC & \\
\hline$\S$ & LuPME91 & L-E & \\
\hline * & LUPME92 & SA-S & PttPME1 \\
\hline * & $\underline{\text { LUPME102 }}$ & OV; SA-S & \\
\hline$¥$ & LuPME103 & O-X; EC-LC & \\
\hline$¥$ & LuPME105 & O-X; EC-LC & \\
\hline$\S$ & LuPMEI1 & O-X; LC-EC & \\
\hline
\end{tabular}

Table 4 Genes putatively associated with fiber development during elongation, thickening, and maturation processes (Continued)

\begin{tabular}{|c|c|c|}
\hline$\S$ & LUPMEl17 & LC-EC \\
\hline$\S$ & LUPMEI19 & LC-EC \\
\hline$\S$ & LUPMEI23 & O-X \\
\hline$\S$ & LUPMEI24 & LC-EC \\
\hline$\S$ & LUPMEI25 & LC-EC \\
\hline * & LUPMEI27 & SA-S \\
\hline$\S$ & LUPMEI28 & LC-EC \\
\hline$¥$ & LUPMEI29 & EC-LC \\
\hline$\S$ & LUPMEI38 & $L-E$ \\
\hline$\S$ & LUPMEI40 & O-X \\
\hline$\S$ & LUPMEI41 & O-X; L-E; LC-EC \\
\hline$¥$ & LUPMEI43 & EC-LC \\
\hline$¥$ & LUPMEI44 & $E-L$ \\
\hline * & LUPMEI45 & SA-S \\
\hline$\S$ & LUPMEI51 & L-E; LC-EC \\
\hline$¥$ & LUPMEI54 & O-X; EC-LC \\
\hline$\S$ & LuPMEI55 & OV \\
\hline$\S$ & LuPMEI56 & O-X; L-E \\
\hline * & LuPMEI59 & SA-S \\
\hline$\S$ & LuPMEI63 and LuPMEI68 & $O-X ; L-E$ \\
\hline$¥$ & LuPMEI65 & O-X; E-L; EC-LC \\
\hline$¥$ & LuPMEI66 & $E-L$ \\
\hline$\S$ & LuPMEI68 & O-X \\
\hline$\S$ & LUPMEI71 & $0-X$ \\
\hline$¥$ & LUPMEI72 & EC-LC \\
\hline$\S$ & LUPMEI74 & $L-E$ \\
\hline$¥$ & LUPMEI76 & EC-LC \\
\hline$\S$ & LUPMEI82 & $L-E$ \\
\hline$\S$ & LuPMEI83 & OV \\
\hline$¥$ & LuPMEI85 & O-X; E-L \\
\hline$\S$ & LuPMEl86 & $L-E$ \\
\hline$\S$ & LuPMEI87 & O-X \\
\hline$¥$ & LuPMEl89 & $E-L$ \\
\hline$\S$ & LuPMEI90 & O-X; L-E; LC-EC \\
\hline$\S$ & LuPMEI94 & L-E \\
\hline$\S$ & LuPMEI95 & L-E \\
\hline \multicolumn{3}{|c|}{$\begin{array}{l}\text { Genes putatively associated with specific fiber development processes are marked } \\
\text { by symbols: elongation (*), thickening ( } ¥) \text {, and thickening and maturation (§). If } \\
\text { two genes are shown in one line it means the primers used amplified both genes. } \\
\text { (OV) expressed only in vascular tissues; (SA-S) present in SA but not in the rest of } \\
\text { the stem (EF, LF, ECP, LCP); (O-X) present in outer tissues (cortical peel and fibers) } \\
\text { but not xylem; (L-E) present in LF but not EF; (E-L) present in EF but not LF; (EC-LC) } \\
\text { present in ECP but not LCP; (LC-EC) present in LCP but not ECP; (SA) only present } \\
\text { in SA; (LCP) only present in LCP. The genes underlined were found to be "SA-S" } \\
\text { using the qRT-PCR data, however, according to RNAseq assembled reads they are } \\
\text { present in other parts of the stem in addition to SA. As they were only detected in } \\
\text { SA with qRT-PCR it is expected that their expression is higher in the SA. }\end{array}$} \\
\hline
\end{tabular}




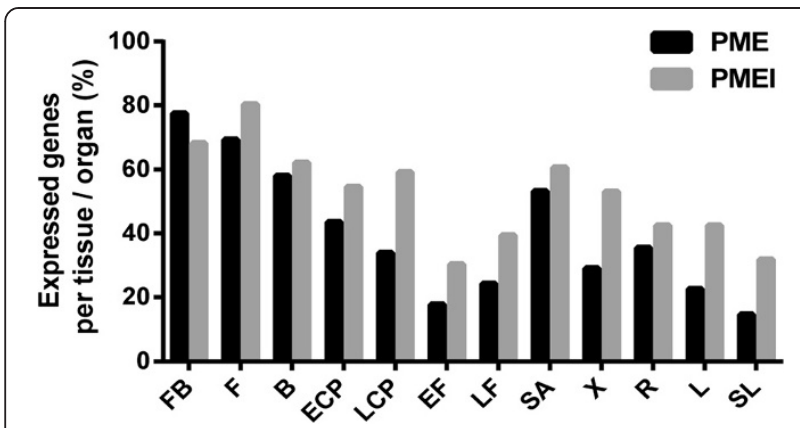

Figure 3 Percentage of genes detected by qRT-PCR per tissue. The percentage was calculated based on those genes that showed transcript expression in at least one tissue.

of the PMEIs is low at early stages of fiber development (i.e. EF stage), when fibers actively synthesize secondary cell walls, and demethylesterification of the newly synthesized homogalacturonan is required. However, when the cell wall deposition ceases, in the late fiber stage, PMEIs are expressed, and so the PME activity diminishes. Seven PMEs and three PMEI were expressed in the shoot apex (SA), but not in any other of the stem vascular tissues. Moreover, nine PMEs and six PMEIs were expressed in the early cortical (ECP) peel, but not the late cortical peel (LCP), and three PMEs and nine PMEIs were expressed in late cortical peel but not early cortical peel (Figures 4A and 5A). 13 PMEs and 14 PMEIs were found only in reproductive tissues; and three PMEs and two PMEI were found only in vascular tissues (Figures 4B and 5B). Seven PMEs and six PMEIs showed specific transcript expression in only one tissue/organ; these transcripts were detected in flower buds (four PMEs and two PMEIs), flowers (one PMEI), bolls (one of each), xylem (one PMEI), roots (one PMEI), late cortical peel (one PME), and shoot apex (one PME). Two of these might be important for phloem fiber development: LuPME71, which was detected only in LCP, a fiber containing tissue where secondary cell wall deposition and maturation is taking place, and LuPME102, only detected in the SA, where intrusive growth takes place (Figure 2A-B).

Previous publications have reported transcript expression patterns of specific LuPMEs. Al-Qsous et al. [13] found that the transcript abundance of LuPME5 is higher than either LuPME1 or LuPME3 in hypocotyls. Our results were consistent with these observations: The calculated delta- $\mathrm{C}_{\mathrm{T}}$ of LuPME5 was higher than LuPME1 or LuPME3 in all the tissues tested (Figure 2). Our transcript abundance data also showed that LuPME5 was expressed in the shoot apex, while LuPME3 was not, which could be correlated with the findings that showed that LuPME5 transcript abundance was higher in the upper parts of the hypocotyl after two days of growth, while LuPME3 was higher in the bottom of the hypocotyl [13]. Mareck et al. [14] found a very high transcript abundance of LuPME3 in roots, as observed with the promoter fusion in tobacco [11], in which a GUS construct using LuPME3 promoter was used to detect its expression in stems, roots and leaves. The expression was observed in the vascular tissues of roots, shoots and young leaves. This correlates with our results, as we detected LuPME3 transcript expression only in roots, late fibers and the boll. Our study used mature leaves, rather than young leaves, which may explain why we failed to detect transcript expression in this tissue, in contrast to Mareck et al.

\section{Protein subcellular localization}

To be secreted to the cell wall via the Golgi apparatus and secretory pathway, PMEs and PMEIs require an $\mathrm{N}$-terminal signal peptide or a transmembrane domain [4]. As shown in Figure 6, we found that 71/105 LuPMEs had a predicted transmembrane domain and/or signal peptide, and 81/95 LuPMEIs had a transmembrane domain and/or signal peptide (Tables 1, 2 and 3). To further investigate subcellular localization, we used WoLF PSORT and Plant-mPLoc [23,24]. Using Wolf PSORT we
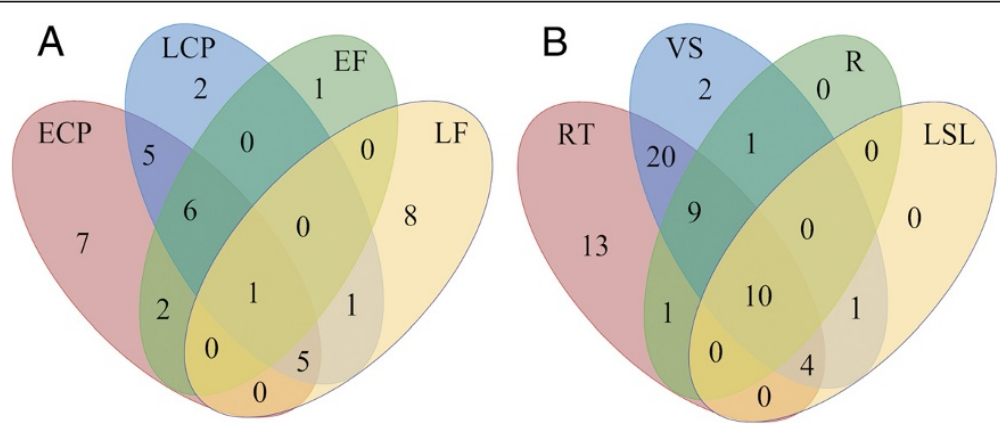

Figure 4 LuPME transcript expression in various tissues. Venn diagram showing the number of LuPMEs detected in phloem-fiber containing tissues (A) and in tissue systems (B) ECP: early cortical peels. LCP: late cortical peels. EF: early fibers. LF: late fibers. RT: reproductive tissues. VS: Vascular tissues at shoot. R: Root. LSL: Leaves and senescent leaves. 

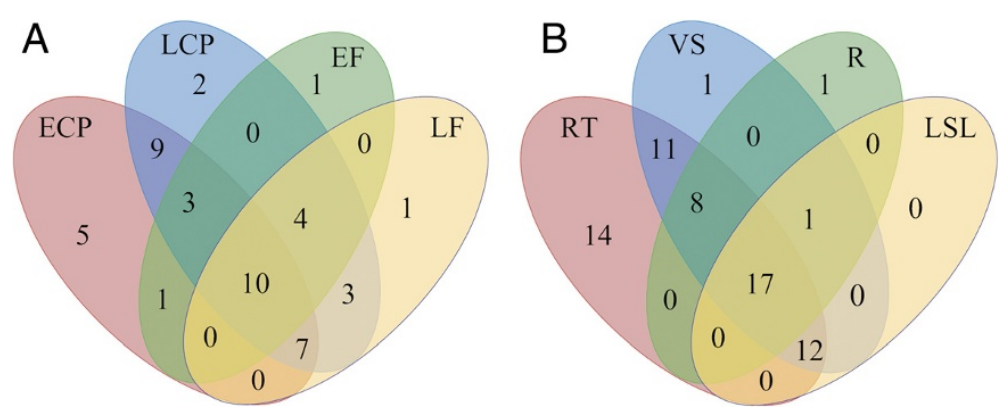

Figure 5 LuPMEl transcript expression in various tissues. Venn diagram showing the number of LuPMEls detected in phloem-fiber containing tissues (A) and in tissue systems (B) ECP: early cortical peels. LCP: late cortical peels. EF: early fibers. LF: late fibers. RT: reproductive tissues. VS: Vascular tissues at shoot. R: Root. LSL: Leaves and senescent leaves.

found 56/105 LuPMEs, and 71/95 LuPMEIs that were predicted to be secreted to either the cell wall or to the plasma membrane. Plant-mPLoc predicted 104/105 LuPMEs to be secreted to the cell wall, and 88/95 LuPMEIs were predicted to be secreted to the plasma membrane or the cell wall. In total all the LuPMEs and 93/95 LuPMEIs were predicted to be extracellular, based on protein subcellular localization software prediction and/or the presence of signal peptide and/or transmembrane domain. The two LuPMEIs, LuPMEI11 and LuPMEI46 that were not predicted to be extracellular were predicted by both software tools to be localized to the nucleus.

\section{Cleavage site}

During their maturation, most Type-1 PMEs are proteolytically cleaved at one of two possible sites between the PMEI domain and the PME domain, before exiting the Golgi apparatus. These sites are designated binding motif 1 (BM1), and binding motif 2 (BM2) (Figure 1), and are separated by between 11-32 amino acids in Arabidopsis [8]. A conserved cleavage site consisting of four residues with the pattern [RKQ][RKEHLN][LDMI] [LMAKR] was previously defined by analysis of $A$. thaliana PMEs $[4,8]$. We identified this pattern at a single site in each of $25 / 60$ of the predicted flax Type-1 PMEs. Moreover, 19/60 flax Type-1 PMEs had two sites that matched the previously defined pattern, and these were separated by between 14 and 33 residues. In 3/60 of the LuPMEs, a novel tetrapeptide motif (RRKL or GRLL) was found in the place of the conserved pattern in BM2. Other novel motifs were also found, but these were all accompanied by a conserved motif in the other binding site, we found RKVA and RRLW in BM1, and REYL and RRFL in BM2. In 13/60 Type-1 PMEs, a cleavage site motif was not found (Table 1). Figure 7 shows the distribution of sizes of the mature proteins of PMEs and PMEIs (after signal peptide and/or pro-region removal, if present).

There was no significant difference (Fisher's Exact Test $p>0.05)$ in the distribution of the cleavage site features between the 45/60 predicted PMEs for which evidence of transcription has been found, and the $15 / 60$ predicted PMEs for which no evidence has been found. The inability to cleave out the PMEI domain (pro-region) would presumably prevent the export of the PMEs to the cell wall, according to Wolf et al. [8], who showed that unprocessed Type-1PMEs are retained in the Golgi apparatus. Nevertheless, LuPME5 was detected in both the unprocessed and processed forms in cell walls of flax cell cultures [13], and LuPME3 was only detected in
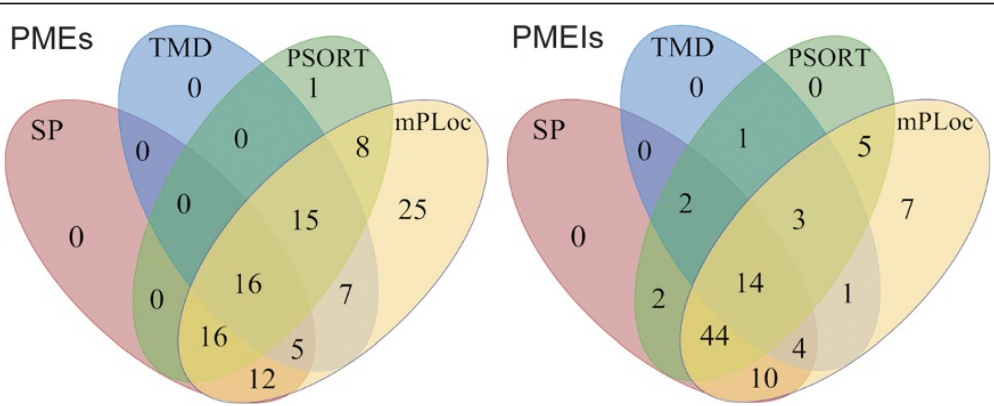

Figure 6 Subcellular localization of PMEs and PMEls. Number of PMEs and PMEls with transmembrane domains, signal peptides, and predicted to be secreted to the cell wall or plasma membrane using WoIF PSORT and Plant-mPLoc. 


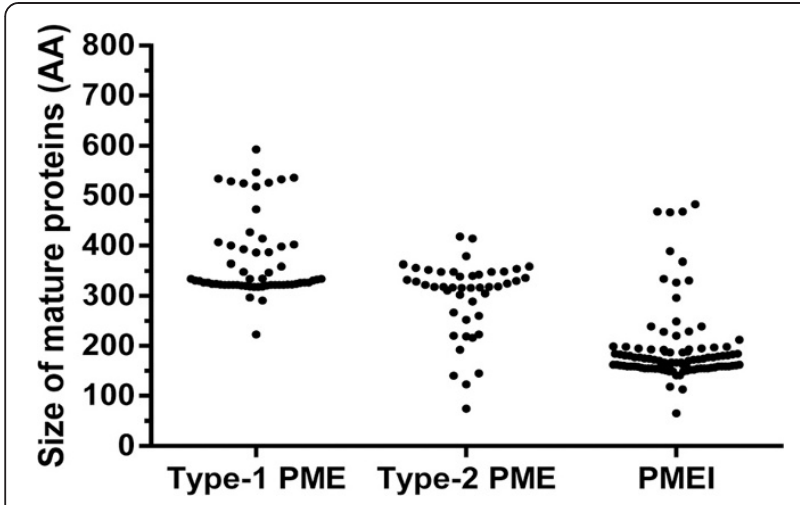

Figure 7 Size of mature proteins. The signal peptide and/or cleavage site, if present, were removed.

the unprocessed form in flax seedlings and callus [14]. This raises the possibility that the processing of Type-1 LuPMEs might be dispensable for the proper functioning or at least localization of the protein.

\section{Isoelectric point}

The isoelectric point is one of the factors that influences the action of PMEs (i.e. random, acidic pI, or blockwise, alkaline pI [37]) and so can facilitate either stiffening or loosening of the cell wall. Consequently, the prediction of the pI of the proteins contributes to the definition of their physiological role in the plant. We calculated the pI for the mature proteins (i.e. with any signal peptide or pro-region removed) (Figure 8). Most of the PMEIs (51) had an acidic pI, while 26 had a basic pI ( $\mathrm{pH} \overline{\mathrm{x}}: 6.48$, $\left.\sigma_{\mathrm{x}}: 1.77.\right)$. On the other hand, most of the PMEs (70) had a basic (above pH8.0) pI (pH $\overline{\mathrm{x}}:$ 8.26, $\sigma_{\mathrm{x}}$ : 1.46.), while just a few (13) had an acidic pI, below 6: Out of these, four showed tissue specificity: Two in FB: LuPME93 and LuPME37, and two in fiber containing tissues: LCP (LuPME71), and SA (LuPME102). As an acidic pI would

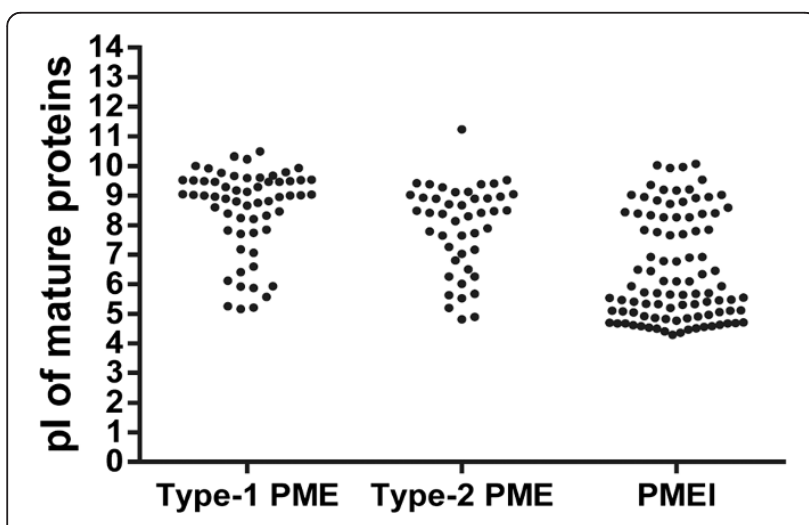

Figure $\mathbf{8}$ Isoelectric point of mature proteins. The signal peptide and/or cleavage site, if present, were removed. lead to random demethylesterification, which ultimately could lead to cell wall loosening; it could be expected that when LuPME71 is expressed in the LCP, it decreases the rigidity of the connections between fibers, while LuPME102 might enhance fiber growth as it could loosen the connections between parenchyma cells to facilitate fiber intrusive growth. Only one of the genes whose transcript expression was detected in the EF or LF had an alkaline pI, this was LuPME66 (pI 5.87), which was expressed in reproductive tissues, xylem, LCP and LF. This could have a role similar to the described for LuPME71.

The wide range of predicted isoelectric points for the mature PMEs (pH 4.75 to 11.25; Tables 1 and 2) is consistent with previous reports from flax. Gaffe et al. [38] tested the PME activity from cell wall of flax calli; they found isoforms at pI 5.5, 7, 7.3, 7.8, 8.8, and 10 . Mareck et al. [39] found a similar range of isoforms in flax calli; they found PMEs with pIs 4.3, 4.8, 6.3, 7.1, 7.6, and 9.6. Alexandre et al. [40] found 2 PMEs in hypocotyls, at pH 8 to 9 , and at $\mathrm{pH} 9.5$ to 10 . Al-Qsous et al. [13] observed PME activity in the hypocotyls at 5 different isoelectric points, from $\mathrm{pH} 7.0$ to 10.0. Finally, Mareck et al. [14] also found a similar pattern of the isoforms in epicotyls, cotyledons, hypocotyls and roots; they found two neutral, four basic and one strongly basic PME isoform.

\section{Phylogenetic analysis}

To classify the predicted LuPMEs and LuPMEIs on the basis of amino acid sequence similarity and inferred evolutionary relationships, we aligned their amino acid sequences with predicted PMEs and PMEIs from four other angiosperms: cassava (Manihot esculenta), castor (Ricinus communis), poplar (Populus trichocarpa), and A. thaliana. These species were chosen because Arabidopsis is a well-characterized model organism, and castor, cassava, and poplar are in the same taxonomic order (Malpighiales) as flax, and whole-genome assemblies are available for each of these species. Following alignment, maximum likelihood phylogenetic trees for PMEs (Figure 9) and PMEIs (Figure 10) were constructed. Based on the groups defined by Louvet et al. [41] for Arabidopsis PMEs, the branch length, and the bootstrap values (Additional file 4: Figure S2), six major monophyletic groups of PME could be defined (A, B, C, D, E, and $\mathrm{F}$ which correspond to groups $3,1,2,1,4$, and 1 respectively in Louvet et al. [41]). Group A included five LuPMEs; three of them were Type-1 PME, and none of them had a cleavage recognition site. The PMEs in all the organisms of group B were Type- 1 PMEs, and all the LuPMEs in this group had a cleavage recognition site, and a transmembrane domain, but no predicted signal peptide. Group C was composed of Type-1 and 


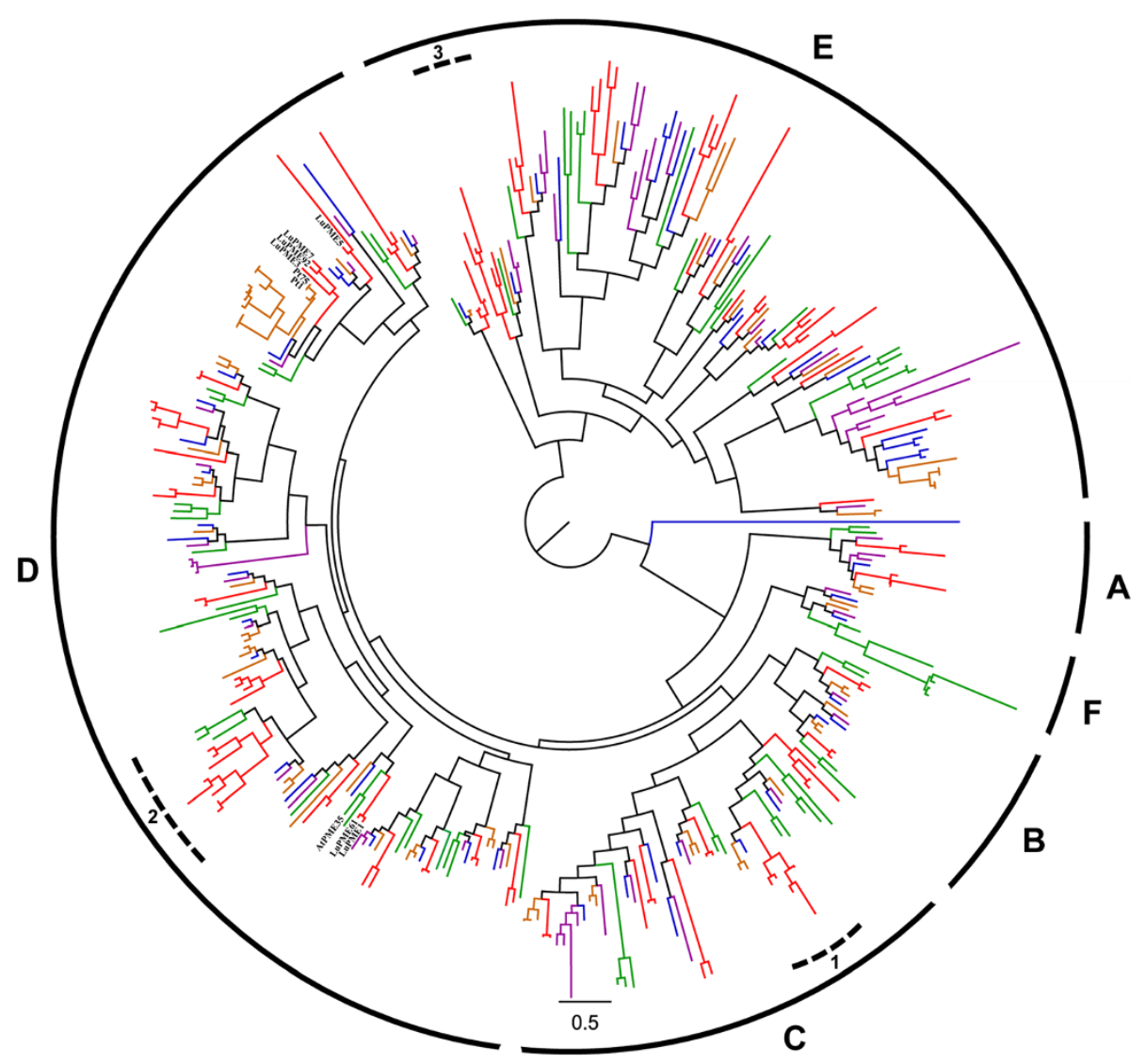

Figure 9 Maximum likelihood dendrogram of PMEs in flax and related species. The main groups (labeled $\mathbf{A}-\mathbf{F}$ ) and some subgroups are shown. The previously reported LuPMEs and the homologous LuPMEs to PttPME1 and AtPME35 are labeled. Red: Linum usitatissimum; Purple: Manihot esculenta; Blue: Ricinus communis; Orange: Populus trichocarpa; Green: Arabidopsis thaliana. 100 bootstraps and 2 search-replicates (bootstrap values shown in Additional file 4: Figure S2).

Type-2 PMEs. All of the Type-1 PMEs had a cleavage recognition site, and none of the Type-2 PMEs had either a signal peptide or transmembrane domain. Group D contained the previously described LuPME1, LuPME3, and LuPME5, we did not find any characteristic defining this group based on the parameters we described above (e.g. Table 1). The PMEs of all the species in group E were Type-2 PMEs. Finally group F contained PMEs of all the species analyzed except flax.

In the PMEI phylogenetic tree (Figure 10), groups were distinguished by very low bootstrap values in the base nodes (Additional file 5: Figure S3), making subclassification of PMEIs ambiguous. Furthermore, we did not find any common sequence features that distinguished subgroups of PMEIs from each other.

Phylogenetic analysis of LuPMEs and LuPMEIs grouped 43 pairs of LuPMEs (out of 105 genes in total) and 39 pairs of LuPMEIs (out of 95 genes in total) at the terminal nodes of the tree (Figures 9 and 10). The remaining genes, 19 PMEs and 17 PMEIs, did not have obvious paralogs. The pairs of genes were confirmed by reciprocal BLASTn and BLASTp to test if they were the best BLAST match. 38/43 pair of LuPMEs and 38/39 pairs of LuPMEIs were found to be the best match to one another. This suggests these probably originated from a recent whole genome duplication event believed to have occurred 5 to 9 MYA [15]. Indeed, for the LuPMEIs, the estimated time of divergence of presumptive paralogs was calculated to be 4.5-8.4 MYA, and for the LuPMEs estimate was 6.4 -11.9 MYA (Additional file 6: Table S2). Lineage-specific expansion of groups of PMEs or PMEIs may indicate that selection had occurred for particular functions in flax. Expansion of at least three sub-groups $(\mathrm{C} 1, \mathrm{D} 2$, and E3) of PMEs was evident in the ML tree (Figure 9).

We identified PMEs genes that have been associated with stem development in previous studies in other species, and found their presumptive homologs in flax. Siedlecka et al. [42] found that when the transcript abundance of PttPME1 (accession no. AJ277547) in hybrid Aspen (Populus tremula $\times$ tremuloides) increased, the fiber elongation decreased, and conversely, when the transcript abundance of the gene was low it stimulated fiber elongation. The 


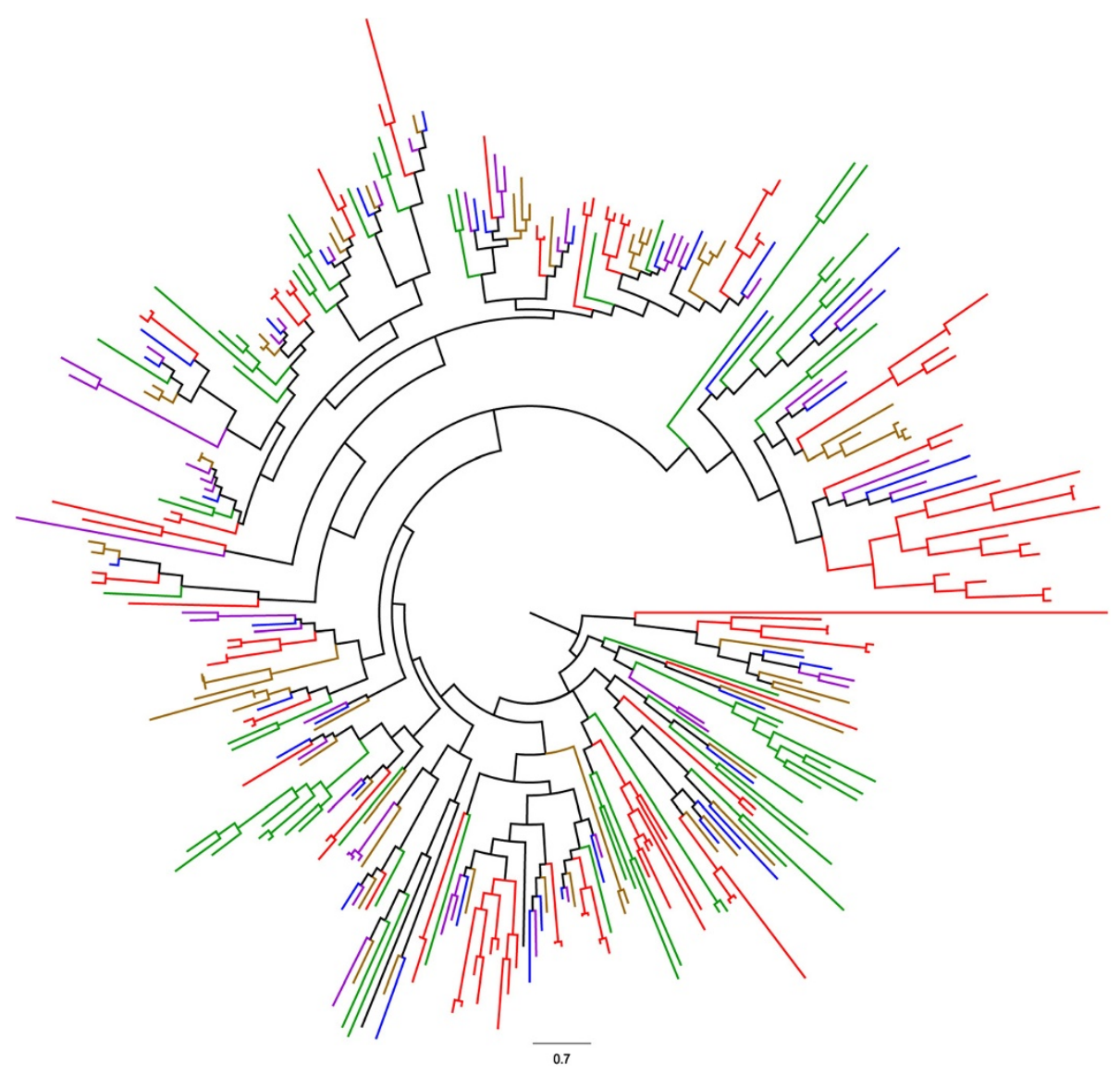

Figure 10 Maximum likelihood dendrogram of PMEls in flax and related species. Red: Linum usitatissimum; Purple: Manihot esculenta; Blue: Ricinus communis; Orange: Populus trichocarpa; Green: Arabidopsis thaliana. 100 bootstraps and 2 search-replicates (bootstrap values shown in Additional file 5: Figure S3).

closest PMEs in P. trichocarpa for PttPME1 are Pt1 (POPTR_0001s16250.1) and Pt75 (POPTR_0214s00200.1). Based on the phylogenetic tree, we found three LuPMEs that were closely related to Pt1and Pt75; they were LuPME7, LuPME92, and LuPME3 (Figure 9; Table 1), which were all type-1 PME, as PttPME1. The study of these genes will be important in future studies as they may regulate fiber elongation the same way as in poplar, as the fibers of both plants elongate intrusively. Hongo et al. [43] found that the type-1 PME AtPME35 (At3g59010), has a role in strengthening the inflorescence stem of Arabidopsis, by mediating the demethylesterification of the primary cell wall of cortical cells and interfascicular fibers, this gene was suggested to have a blockwise demethylesterification action. We inferred that the common ancestor of LuPME61 and LuPME1 is the likely ortholog to AtPME35 (Figure 9; Table 4). Both LuPME61 and LuPME1 have basic pIs (9.42 and 8.82, respectively), similar to the pI of the mature protein of AtPME35 (pI 8.70), so it is possible that they also have a blockwise demethylesterification activity similar to AtPME35, which leads to stiffening of the cell wall. The study of loss-offunction mutants for these genes in flax might identify informative phenotypes related to stem development.

\section{Conserved residues in PMEs}

We searched the predicted LuPMEs for conserved amino acid residues previously reported to be important for PME function. These amino acids were identified in a Type-1 PME from tomato (Solanum lycopersicum), (PME1_SOLLC, SwissProt P14280), and the positions listed here refer to that sequence [7]. Three residues are proposed to be catalytic residues: D132, D153, and R221. Two residues, Q109 and Q131, are believed to stabilize the intermediate that is formed after nucleophilic attack on the carboxylmethyl group. Finally, six aromatic amino acids at conserved positions are required for substrate binding (F80, Y135, F156, Y218, W223, and W248), and of these F80, Y135, and W223 are possible targets of the PMEIs. We searched the predicted LuPMEs for all eleven of the residues that have been proposed to be critical to PME function (Tables 1 and 2). The most highly conserved 
residues were the catalytic residues: D132, D153, and R221, which could be identified in (91/105), (96/105), and $(97 / 105)$ of the predicted LuPMEs, respectively (Figure 11A). The aromatic residues responsible for substrate binding were also highly conserved, where any aromatic residue is considered to be a conserved residue in comparison to the substrate binding positions defined in PME1_SOLLC (i.e. F80, Y135, F156, Y218, W223, and W248) . The most highly conserved of these were W223 (93/105) and W248 (92/105), and the conservation of other substrate binding residues was also high: F80 (74/105), Y135 (77/105), F156 (89/105), and Y218 (89/105). Among these aromatic residues there were three positions (F156, W223 and W248) in which the identity (and not merely similarity) of the aromatic amino acids was also highly conserved: at F156, F was found in $88 / 89$ of the LuPMEs that had an aromatic residues at that position; at W223, 90/93 aromatic residues were W, and in W248 91/92 aromatic residues were W. F80, Y135, and W223 are responsible of substrate binding of the PMEs and also interact with the PMEI. F80 generated the highest number of contacts (17 in total) with the PMEI [7]. Out of all the aromatic residues, position F80 was the least conserved, followed by Y135, meanwhile W223 showed the highest conservation. This might imply that F80 and Y135 are not fundamental for binding to the substrate, as it might be W223, and on the other hand, lacking these residues may limit inhibition by the PMEIs.

\section{Conserved residues PMEI}

PMEIs have four antiparalell alpha-helices $(\alpha 1, \alpha 2, \alpha 3$ and $\alpha 4$ ) arranged in an up and down topology, and three short alpha-helices ( $\alpha \mathrm{a}, \alpha \mathrm{b}$, and $\alpha \mathrm{c})$ at the N-terminus [7]. Two groups have studied PMEI structure in detail. Di Matteo et al. [7] identified residues with important roles in the structure and activity of a PMEI from kiwi (Actinidia deliciosa; PMEI_ACTDE, SwissProt accession P83326), focusing on the interaction with a PME. They identified cysteine residues that generated disulfide bridges conecting helices $\alpha \mathrm{a}$ and $\alpha b$ (C9 and C18), and helices $\alpha 2$ and $\alpha 3$ (C74 and C114). Furthermore, PMEI residues T73, E76, N77, T113, and D116 were found to allow interaction of the PMEI with three of the aromatic residues of the PME (F80, Y135, and W223). An acidic patch was formed by three conserved residues on both the $\alpha 2$ helix (E76, D80, and D83) and on the $\alpha 3$ helix (D96, D109, and D116). Finally, salt bridges occurred between the PMEI residues D116 and E76 and PME residues $\mathrm{K} 224$ and $\mathrm{R} 81$. Hothorn et al. [6] also studied the important residues for the PMEI activity and structure, using Arabidopsis PMEI-1 (PMEI1_ARATH, SwissProt accession number Q9LNF2) as a model. They identified a disulfide bridge connecting helices 5 and 6 ( $\alpha 2$ and $\alpha 3$ in Di Matteo et al. [7]), formed by residues C71 and C111. They also identified a residue responsible for the $\mathrm{N}$-terminal orientation (P28) that is located between the three $\mathrm{N}$-terminal $\alpha$-helices and the four $\alpha$-helices towards the $\mathrm{C}$-terminal; and several residues contributing to the bundle-hairpin interface.

We searched the predicted PMEIs of flax for the critical residues identified by Di Matteo et al. [7] and Hothorn et al. [6]. Our results (Table 3) are presented using as reference positions the mature PMEI_ACTDE protein. The residues in LuPMEIs with the highest conservation were C74 and C114 (both 94/95), which generate a
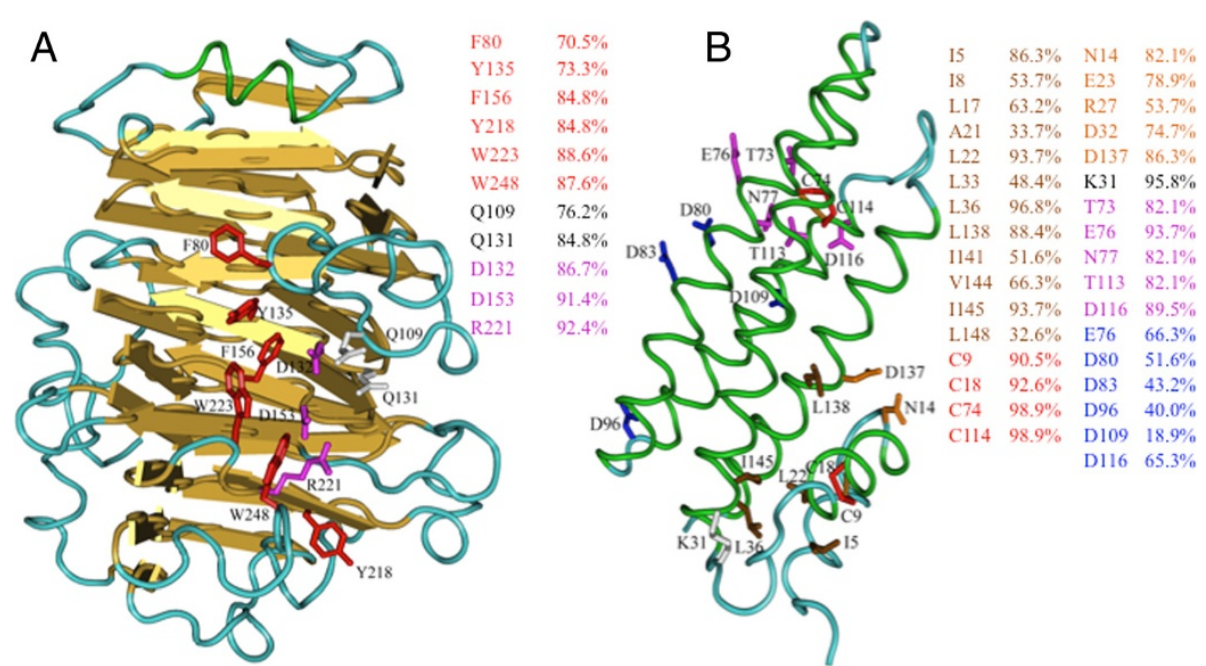

Figure 11 Tertiary structures showing the conserved residues analyzed in relation to the reported structures. Structures shown are for PME1_SOLLC (A) and PMEI_ACTDE (B), PDB: 1XG2. For the residues involved in bundle-hairpin interface in PMEl_ACTDE only those residues with conservation higher than $80 \%$ are labeled in the structure. 
disulfide bridge (Figure 11B). The conservation of the other two cysteines, C9 and C18, which stabilize the protein by hydrophobic interactions, was slightly lower, (86/95) and (88/95), respectively. The conservation (i.e. similarity, not necessarily identity) in the polar PMEI residues that interact with the aromatic PME residues, F80, Y135, and W223, was higher than $80 \%$ in all the cases: T73 (78/95), E76 (89/95), N77 (78/95), T113 (78/95), and D116 (85/95). 87.4\% (83/95) of the PMEIs had conservation of at least 3 out of the 5 polar residues. On the other hand, the Aspartic acid (D), and Glutamic acid (E) residues that are predicted to be important in the generation of an acidic patch on alpha helices 2 and 3, had a low conservation (i.e. similarity, not necessarily identity), $47.5 \%$ on average. The conservation of the residues contributing to the bundle-hairpin interface was also low. Out of the 12 non-polar residues analyzed, only five were conserved (i.e. similarity, not necessarily identity) in more than $80 \%$ of the LuPMEIs, those are I5 (82/95), L22 (89/95), L36 (92/95), L138 (84/95), and I145 (89/95), the rest (I8, L17, A21, L33, I141, V144, and L148) were below $70 \%$ of conservation.

\section{Gene expression and conserved residues}

We tested whether there was any correlation between the transcript expression evidence we obtained and the presence of the critical residues described above (Tables 1, 2 and 3). In general, genes that are not expressed may accumulate mutations, including mutations in residues critical to the normal function of the protein. We found that when we analyzed the 33 critical residues in the LuPMEIs as a group, the conservation of these residues among the expressed genes (73.2\%) was significantly higher than in the genes for which transcript expression was not detected $(63.9 \%)$ (Fisher's Exact Test, $\mathrm{p}<0.05)$. Taking together the 11 critical residues studied in the LuPMEs, we found that in the expressed genes, the level of conservation (86.8\%) was significantly higher than the conservation observed in the non-expressed genes (75.3\%) (Fisher's Exact Test, $\mathrm{p}<0.05$ ). However when we individually analyzed the residues we found that in the LuPMEs only three out of the 11 residues (Q109, F156, and R221) showed significantly higher conservation in the expressed genes when compared to the genes without evidence of transcript expression (Fisher's Exact Test, $\mathrm{p}<0.05)$. In the remaining eight there was no significant difference (Fisher's Exact Test, $\mathrm{p}>0.05$ ). The critical residue that showed the greatest change in conservation in relation to transcript expression was Q109, which was found in $83.1 \%$ of expressed LuPMEs, but only $57.1 \%$ of LuPMEs with no evidence of transcript expression. In the same way, we individually analyzed the LuPMEIs conserved residues, and found that only 5 of the 33 residues (L36, V144, C18, R27, and D83 ) have a significant higher conservation in expressed genes respect to non-expressed genes (Fisher's Exact Test, $\mathrm{p}<0.05$ ). In the remaining 28 residues there was no significant difference (Fisher's Exact Test, $\mathrm{p}>0.05$ ). The two greatest differences were observed in two of the residues contributing to the bundle-hairpin interface: V144 was conserved in $71.1 \%$ of expressed LuPMEs, but only $33.3 \%$ of non expressed genes, and R27 from 59.0\% to $16.7 \%$. Interestingly, the change in conservation was very different between the two pairs of cysteines of the PMEIs, which generate the disulfide bridges, $\mathrm{C} 74$ and $\mathrm{C} 114$ conservation changed both from $98.8 \%$ in expressed genes, to $100 \%$ in both, in non-expressed genes. Conversely, the conservation at positions $\mathrm{C} 9$ and $\mathrm{C} 18$ was reduced drastically, although not significantly in C9, from $92.8 \%$ and $96.4 \%$ in expressed genes, to $75.0 \%$ and $66.7 \%$, respectevely in non expressed genes. This might indicate that there is more evolutionary pressure on residues $\mathrm{C} 74$ and $\mathrm{C} 114$, which indicates that they might be more important for the structure of the protein.

The residue at position K31(respect to PMEI_ACTDE [7]) or P28 (respect to PMEI1_ARATH [6]) has been reported to affect the orientation of the N-terminal of the PMEIs. We found 13 different amino acids at this position. There were three prevalent residues in this position, they were P (19/95), A (18/95), and I (17/95) residues. Hothorn et al. [6], found that when they mutated P28 to Ala in PMEI1_ARATH, the inhibitory activity of the protein diminished. So the 18 PMEIs with alanine could have a decline in activity.

\section{Conclusions}

PMEs, regulated in part by PMEIs, modify cell and tissue properties by demethylesterification of pectins within cell walls and the middle lamella. Flax phloem fibers elongate intrusively by penetrating the middle lamella of the cells below and above them. This process requires the loosening of the middle lamella, the strengthening of the cell wall of the growing fiber, and then the creation of a new contact interface with the cell. In these processes, PMEs might be involved. Here we described 105 putative PMEs and 95 putative PMEIs in the flax genome, of which 77 and 83 genes, respectively, had evidence of transcript expression. The proportion of PMEs and PMEIs in the flax genome as a proportion of all predicted proteins was similar to most other dicots (Additional file 3: Figure S1). We defined a list of candidate genes that could play a role in fiber development (Table 4), their specific transcript expression in fiber containing tissues and mutant of these genes should be analyzed in detailed in future studies, and these genes area target for manipulation through reverse genetics. 


\section{Additional files} Additional file 1: Table S1. List of primers and TaqMan probes used
for GRT-PCR.

Additional file 2: FASTA formatted LuPME and LuPMEI sequences. Additional file 3: Figure S1. Percentage of PMEs and PMEls, respect to the total number of proteins, in Embryophyta plants with available full genomes in Phytozome (version 9.1).

Additional file 4: Figure S2. Maximum likelihood tree of PMEs in Flax and related species. The main groups, and important subgroup are shown. The homologous LuPMEs to PttPME1 and AtPME35 are shown with an arrow. Red: Linum usitatissimum; Purple (Me): Manihot esculenta; Blue (Rc): Ricinus communis; Orange (Pt): Populus trichocarpa; Green: Arabidopsis thaliana. 100 bootstraps and 2 search-replicates.

Additional file 5: Figure S3. Maximum likelihood tree of PMEls in flax and related species. The main groups, and subgroup D1 are shown. Red: Linum usitatissimum; Purple (Me): Manihot esculenta; Blue (Rc): Ricinus communis; Orange (Pt): Populus trichocarpa; Green: Arabidopsis thaliana. 100 bootstraps and 2 search-replicates.

Additional file $\mathbf{6}$ Table S2. Genetic distance between possible paralogs in the LuPMEs and LuPMEIs gene families. The Kimura 2-parameter mode was used to calculate the genetic distance, which was used to calculate the divergence time using $t=K / 2 r$, where $t$ is time, $K$ is the genetic distance, and $r$ is the substitution rate, either $1.5 \times 10^{-8}$ or $8.1 \times 10^{-9}$

\section{Competing interests}

The authors declare that they have no competing interests.

\section{Authors' contributions}

DPL performed all experiments and analyses and wrote the original draft of this manuscript. MKD supervised experiments and analyses and edited the manuscript. Both authors read and approved the final manuscript.

\section{Acknowledgements}

We thank Ran Zhuo for training on the Fluidigm instrument. Funding was provided by Genome Canada and Natural Sciences and Engineering Research Council (Canada)

Received: 3 August 2013 Accepted: 26 October 2013

Published: 30 October 2013

\section{References}

1. Richard L, Qin LX, Goldberg R: Clustered genes within the genome of Arabidopsis thaliana encoding pectin methylesterase-like enzymes. Gene 1996, 170(2):207-211.

2. Cantarel BL, Coutinho PM, Rancurel C, Bernard T, Lombard V, Henrissat B: The Carbohydrate-Active EnZymes database (CAZy): an expert resource for Glycogenomics. Nucleic Acids Res 2009, 37:D233-D238.

3. Micheli F: Pectin methylesterases: cell wall enzymes with important roles in plant physiology. Trends Plant Sci 2001, 6(9):414-419.

4. Pelloux J, Rusterucci C, Mellerowicz EJ: New insights into pectin methylesterase structure and function. Trends Plant Sci 2007, 12(6):267-277.

5. Balestrieri C, Castaldo D, Giovane A, Quagliuolo L, Servillo L: A glycoprotein inhibitor of pectin methylesterase in kiwi fruit (actinidia-chinensis). Eur $J$ Biochem 1990, 193(1):183-187.

6. Hothorn M, Wolf S, Aloy P, Greiner S, Scheffzek K: Structural insights into the target specificity of plant invertase and pectin methylesterase inhibitory proteins. Plant Cell 2004, 16(12):3437-3447.

7. Di Matteo A, Giovane A, Raiola A, Camardella L, Bonivento D, De Lorenzo G, Cervone F, Bellincampi D, Tsernoglou D: Structural basis for the interaction between pectin methylesterase and a specific inhibitor protein. Plant Cell 2005, 17(3):849-858.

8. Wolf $\mathrm{S}$, Rausch T, Greiner S: The N-terminal pro region mediates retention of unprocessed type-I PME in the Golgi apparatus. Plant J 2009, 58(3):361-375

9. Ageeva MV, Petrovska B, Kieft H, Sal'nikov W, Snegireva AV, van Dam JEG, van Veenendaal WLH, Emons AMC, Gorshkova TA, van Lammeren AAM:
Intrusive growth of flax phloem fibers is of intercalary type. Planta 2005 222(4):565-574.

10. Cullis C: Flax. In Oilseeds. 2nd edition. Edited by Kole C. Berlin Heidelberg: Springer; 2007:275-295.

11. Roger D, Lacoux J, Lamblin F, Gaillet D, Dauchel H, Klein D, Balangé AP, David A, Lainé E: Isolation of a flax pectin methylesterase promoter and its expression in transgenic tobacco. Plant Sci 2001, 160(4):713-721.

12. Lacoux J, Klein D, Domon J-M, Burel C, Lamblin F, Alexandre F, Sihachakr D, Roger D, Balangé A-P, David A, et al: Antisense transgenesis of Linum usitatissimum with a pectin methylesterase cDNA. Plant Physiol Biochem 2003, 41(3):241-249.

13. Al-Qsous S, Carpentier E, Klein-Eude D, Burel C, Mareck A, Dauchel H, Gomord V, Balange AP: Identification and isolation of a pectin methylesterase isoform that could be involved in flax cell wall stiffening. Planta 2004, 219(2):369-378.

14. Mareck A, Lamour R, Schaumann A, Chan P, Driouich A, Pelloux J, Lerouge P: Analysis of LuPME3, a pectin methylesterase from Linum usitatissimum, revealed a variability in PME proteolytic maturation. Plant Signal Behav 2012, 7(1):59-61.

15. Wang ZW, Hobson N, Galindo L, Zhu SL, Shi DH, McDill J, Yang LF, Hawkins S, Neutelings G, Datla R, et al: The genome of flax (Linum usitatissimum) assembled de novo from short shotgun sequence reads. Plant J 2012, 72(3):461-473.

16. Punta M, Coggill PC, Eberhardt RY, Mistry J, Tate J, Boursnell C, Pang N, Forslund K, Ceric G, Clements J, et al: The Pfam protein families database. Nucleic Acids Res 2012, 40(D1):D290-D301.

17. Lamesch P, Berardini TZ, Li DH, Swarbreck D, Wilks C, Sasidharan R, Muller R, Dreher K, Alexander DL, Garcia-Hernandez M, et al: The Arabidopsis Information Resource (TAIR): improved gene annotation and new tools. Nucleic Acids Res 2012, 40(D1):D1202-D1210.

18. Stanke M, Morgenstern B: AUGUSTUS: a web server for gene prediction in eukaryotes that allows user-defined constraints. Nucleic Acids Res 2005, 33:W465-W467.

19. Goodstein DM, Shu SQ, Howson R, Neupane R, Hayes RD, Fazo J, Mitros T, Dirks W, Hellsten U, Putnam N, et al: Phytozome: a comparative platform for green plant genomics. Nucleic Acids Res 2012, 40(D1):D1178-D1186.

20. Huis R, Hawkins S, Neutelings G: Selection of reference genes for quantitative gene expression normalization in flax (Linum usitatissimum L.). BMC Plant Biol 2010, 10

21. Petersen TN, Brunak S, von Heijne G, Nielsen H: SignalP 4.0: discriminating signal peptides from transmembrane regions. Nat Methods 2011, 8(10):785-786.

22. Krogh A, Larsson B, von Heijne G, Sonnhammer ELL: Predicting transmembrane protein topology with a hidden Markov model: application to complete genomes. J Mol Biol 2001, 305(3):567-580.

23. Horton P, Park KJ, Obayashi T, Nakai K: Protein subcellular localization prediction with WOLF PSORT. Ser Adv Bioinform 2006, 3:39-48.

24. Chou KC, Shen HB: Plant-mPLoc: a top-down strategy to augment the power for predicting plant protein subcellular localization. Plos ONE 2010, 5(6).

25. Li GQ, Moriyama EN: Vector NTI, a balanced all-in-one sequence analysis suite. Brief Bioinform 2004, 5(4):378-388.

26. Edgar RC: MUSCLE: multiple sequence alignment with high accuracy and high throughput. Nucleic Acids Res 2004, 32(5):1792-1797.

27. Darriba D, Taboada GL, Doallo R, Posada D: ProtTest 3: fast selection of best-fit models of protein evolution. Bioinformatics 2011, 27(8):1164-1165.

28. Zwickl DJ: Genetic algorithm approaches for the phylogenetic analysis of large biological sequence datasets under the maximum likelihood criterion. Austin, Texas: The University of Texas at Austin; 2006.

29. Miller MA, Pfeiffer W, Schwartz T: Creating the CIPRES Science Gateway for Inference of Large Phylogenetic Trees, Proceedings of the Gateway Computing Environments Workshop (GCE): 14 Nov. 2010. New Orleans, LA 2010:1-8.

30. Tamura K, Peterson D, Peterson N, Stecher G, Nei M, Kumar S: MEGA5: Molecular Evolutionary Genetics Analysis using Maximum Likelihood, Evolutionary Distance, and Maximum Parsimony Methods. Mol Biol Evol 2011, 28(10):2731-2739.

31. Kimura M: A simple method for estimating evolutionary rates of base substitutions through comparative studies of nucleotide sequences. J Mol Evol 1980, 16(2):111-120.

32. Koch MA, Haubold B, Mitchell-Olds T: Comparative Evolutionary Analysis of Chalcone Synthase and Alcohol Dehydrogenase Loci in Arabidopsis, 
Arabis, and Related Genera (Brassicaceae). Mol Biol Evol 2000, 17(10):1483-1498.

33. Lynch M, Conery JS: The Evolutionary Fate and Consequences of Duplicate Genes. Science 2000, 290(5494):1151-1155.

34. Arabidopsis $\mathrm{Gl}$ : Analysis of the genome sequence of the flowering plant Arabidopsis thaliana. Nature 2000, 408(6814):796.

35. Fenart S, Ndong YPA, Duarte J, Riviere N, Wilmer J, van Wuytswinkel O, Lucau A, Cariou E, Neutelings G, Gutierrez L, et al: Development and validation of a flax (Linum usitatissimum L.) gene expression oligo microarray. BMC Genomics 2010, 11.

36. Venglat P, Xiang DQ, Qiu SQ, Stone SL, Tibiche C, Cram D, Alting-Mees M, Nowak J, Cloutier S, Deyholos M, et al: Gene expression analysis of flax seed development. BMC Plant Biol 2011, 11.

37. Willats WGT, Orfila C, Limberg G, Buchholt HC, van Alebeek GJWM, Voragen AGJ, Marcus SE, Christensen TMIE, Mikkelsen JD, Murray BS, et al: Modulation of the degree and pattern of methyl-esterification of pectic homogalacturonan in plant cell walls - Implications for pectin methyl esterase action, matrix properties, and cell adhesion. J Biol Chem 2001, 276(22):19404-19413.

38. Gaffe J, Morvan C, Jauneau A, Demarty M: Partial-purification of flax cell-wall pectin methylesterase. Phytochemistry 1992, 31(3):761-765.

39. Mareck A, Gaffé J, Morvan O, Alexandre C, Morvan C: Characterization of isoforms of pectin methylesterase of linum usitatissimum using polyclonal antibodies. Plant Cell Physiol 1995, 36(3):409-417.

40. Alexandre F, Morvan O, Gaffe J, Mareck A, Jauneau A, Dauchel H, Balange AP, Morvan C: Pectin methylesterase pattern in flax seedlings during their development. Plant Physiol Biochem 1997, 35(6):427-436.

41. Louvet R, Cavel E, Gutierrez L, Guenin S, Roger D, Gillet F, Guerineau F, Pelloux J: Comprehensive expression profiling of the pectin methylesterase gene family during silique development in Arabidopsis thaliana. Planta 2006, 224(4):782-791.

42. Siedlecka A, Wiklund S, Peronne MA, Micheli F, Lesniewska J, Sethson I, Edlund U, Richard L, Sundberg B, Mellerowicz EJ: Pectin methyl esterase inhibits intrusive and symplastic cell growth in developing wood cells of Populus. Plant Physiol 2008, 146(2):554-565.

43. Hongo S, Sato K, Yokoyama R, Nishitani K: Demethylesterification of the primary wall by PECTIN METHYLESTERASE35 provides mechanical support to the arabidopsis stem. Plant Cell 2012, 24(6):2624-2634.

doi:10.1186/1471-2164-14-742

Cite this article as: Pinzón-Latorre and Deyholos: Characterization and transcript profiling of the pectin methylesterase (PME) and pectin methylesterase inhibitor (PMEI) gene families in flax (Linum usitatissimum). BMC Genomics 2013 14:742.

\section{Submit your next manuscript to BioMed Central and take full advantage of:}

- Convenient online submission

- Thorough peer review

- No space constraints or color figure charges

- Immediate publication on acceptance

- Inclusion in PubMed, CAS, Scopus and Google Scholar

- Research which is freely available for redistribution

Submit your manuscript at www.biomedcentral.com/submit
Biomed Central 